\title{
Synthesis, Reactions and Antimicrobial Activity of Some New 3-Substituted Indole Derivatives
}

\author{
Asmaa S. Salman', Naema A. Mahmoud1, Anhar Abdel-Aziem1, Mona A. Mohamed², \\ Doaa M. Elsisi ${ }^{1}$ \\ ${ }^{1}$ Department of Chemistry, Faculty of Science, Al-Azhar University, Girls' Branch, Nasr City, Cairo, Egypt \\ ${ }^{2}$ Biochemistry Division, Faculty of Science, Al-Azhar University, Girls' Branch, Nasr City, Cairo, Egypt \\ Email: salman 2007 ok@yahoo.com
}

Received 7 April 2015; accepted 7 June 2015; published 10 June 2015

Copyright (C) 2015 by authors and Scientific Research Publishing Inc.

This work is licensed under the Creative Commons Attribution International License (CC BY).

http://creativecommons.org/licenses/by/4.0/

c) (i) Open Access

\begin{abstract}
Reaction of indole-3-carboxaldehydes 4 with hydrazine derivatives and different substituted acid hydrazides afforded the corresponding hydrazine derivatives 5 a-c and acid hydrazide derivatives 7-11 respectively. Condensation of indole-3-carboxaldehydes 4 with phenacyl bromide and thiourea gives 1,3-thiazol-2-amine derivative 18. On the other hand, reaction 4 with 3-acetylchromene-2one afforded chalcone derivative 19. Compound 4 undergoing Knoevenagel condensation with cyanoacetamide, ethyl cyanoacetate, benzimidazol-2-ylacetonitrile, rhodanine-3-acetic acid, 2,3dihydropyrimidin-4-one derivative and 2,4-dihydropyrazol-3-one afforded the compounds 20a,b, $22,23,27$ and 28 respectively. The structure of the newly synthesized compounds has been confirmed by elemental analysis and spectra data. The antimicrobial activities of the some newly synthesized compounds were measured and showed that most of them have high activities.
\end{abstract}

\section{Keywords}

Indole-3-Carboxaldehyde, Acid Hydrazide, 1,3-Thiazole, Pyrimidine, Antimicrobial

\section{Introduction}

In the recent past, bacterial infections have increased at an alarming rate causing deadly diseases and widespread epidemics in humans. All types of bacterial diseases have taken a high toll on humanity. The resistance of antibiotics to control emerging and pre-emerging bacterial pathogens focused the medicinal chemists to search potential new antimicrobial agents to cure microbial infections effectively [1]. 
Heterocyclic compounds containing nitrogen have been described for their biological activity against various micro-organisms. The indole unit is the key building block for a variety of compounds which have crucial roles in the functions of biologically important molecules. Many indole alkaloids are recognized as one of the rapidly growing groups of marine invertebrate metabolites for their broad spectrum of biological properties [2] [3]. For example, five new indole alkaloids, meridianins A-E have been isolated from the tunicate Aplidium meridianum, which showed cytotoxicity toward murine tumor cell lines [4].

Introduction of different groups to the modified indole structure can produce a series of compounds with multiple activities. Various 3-substituted indoles had been used as starting materials for the synthesis of a number of alkaloids, agrochemicals, pharmaceuticals and perfumes. Also 3-substituted indole derivatives possess various types of broad spectrum's biological activities such as antimicrobial, antitumor, hypoglycemic, anti-inflammatory, analgesic and antipyretic activities [5] [6]. Moreover the substitution at the 3-position of the indole ring can take place by connecting an additional heterocyclic ring, such as imidazole (topsentins, nortopsentins) [7] [8], dihydroimidazole (discodermindole) [9], oxazole (pimprinols A-C, almazole C) [10] [11], thiazole (bacillamide A) [12], quinazoline (tremorgens) [13], and pyrimidine [14]. Therefore, 3-substituted indoles still represent a significant synthetic challenge. In view of the important biological properties of the indole ring, we planned to synthesize a new series of 3-substituted indole derivatives bearing side chains with different structures; as such derivatives could possess interesting and useful antimicrobial activity.

\section{Materials and Methods}

\subsection{Experimental}

Melting points were measured on a Gallenkamp apparatus and are uncorrected. IR spectra were recorded on Shimadzu FT-IR 8101 PC infrared spectrophotometer $\left(v_{\max }\right.$ in $\left.\mathrm{cm}^{-1}\right)$. The $1 \mathrm{H}$ NMR and ${ }^{13} \mathrm{C}$ NMR spectra were determined in DMSO-d6 at $300 \mathrm{MHz}$ on a Varian Mercury VX 300 NMR spectrometer using TMS as an internal standard. Mass spectra were measured on a GCMS-QP1000 EX spectrometer at 70 Ev. Elemental analyses were carried out at the Microanalytical Center of Cairo University. Spectral data of the synthesized compounds were given in Table 1.

\subsubsection{General Procedure for the Synthesis of 2-Substituted-Indole (3a-d)}

Synthesis of 2-substituted- $1 \mathrm{H}$-indole 3a-d was carried out by the procedure of Fischer indole synthesis. Phenylhydrazone derivatives 2a-d were prepared by warming a mixture of compounds 1 a-d $(0.04 \mathrm{~mol})$ and phenyl hydrazine $(0.072 \mathrm{ml}, 0.04 \mathrm{~mol})$ with $60 \mathrm{ml}$ of ethanol and few drops of glacial acetic acid. The resulting reaction mixture was allowed to stirring for about $2 \mathrm{~h}$. The reaction mixture was then poured into ice water $(50 \mathrm{ml})$ where upon the crude compound was precipitated. The residue obtained after filtration was washed with water and used in second step. A mixture of 2a-d $(0.01 \mathrm{~mol})$ and polyphosphoric acid $(20 \mathrm{ml})$ was refluxed for $6 \mathrm{~h}$. After the completion of the reaction, it was filtered and filtrate was poured into ice cooled water. The solid obtained was filtered and recrystallized from the ethanol to give 3a-d.

\section{2-(4-Methylphenyl)-1H-indole $3 a$}

Yellow crystals. Yield: (1.24 g, 60\%); m.p.: 220-221 ${ }^{\circ} \mathrm{C}$. Anal. calcd. for $\mathrm{C}_{15} \mathrm{H}_{13} \mathrm{~N}$ (207.27): C, 86.92; H, 6.32; N, 6.76. Found: C, 86.62; H, 6.12; N, 6.56.

\section{4-(1H-Indol-2-yl)aniline $3 b$}

White crystals. Yield (1.37 g, 66\%); m.p.: 250-252 ${ }^{\circ} \mathrm{C}$. Anal. calcd. for $\mathrm{C}_{14} \mathrm{H}_{12} \mathrm{~N}_{2}$ (208.25):C, 80.74; H, 5.81; N,13.45.Found: C, 80.54; H, 5.61; N, 13.35.

\section{2-(4-Bromophenyl)-1H-indole 3c}

Yellow crystals. Yield (1.63, 60\%); m.p.: 220-222 ${ }^{\circ} \mathrm{C}$. Anal. calcd. for $\mathrm{C}_{14} \mathrm{H}_{10} \mathrm{Br} \mathrm{N}$ (272.14): C, 61.79; $\mathrm{H}$, 3.70; Br, 29.36; N, 5.15. Found: C, 61.59; H, 3.50; Br, 29.16; N, 5.00.

\section{3-(1H-Indol-2-yl)-2H-chromen-2-one 3d}

Dark brown crystals. Yield (1. 57, 60\%; m.p.: 240-242 ${ }^{\circ} \mathrm{C}$ (DMF). Anal. calcd. for $\mathrm{C}_{17} \mathrm{H}_{11} \mathrm{NO}_{2}$ (261.27): C, 78.15; H, 4.24; N, 5.36. Found: C, 78.00; H, 4.04; N, 5.06.

\subsubsection{2-(4-Bromophenyl)-1H-indole-3-carboxaldehyde 4}

Phosphorous oxychloride (21.47 ml, $0.14 \mathrm{~mol}$ ) was added drop wise to $\mathrm{N}, \mathrm{N}^{\prime}$-dimethylformamide (DMF) (10.23 $\mathrm{ml}, 0.14 \mathrm{~mol}$ ) under cooling with an ice bath and the reaction mixture was stirred for $2 \mathrm{~h}$. to prepare the Vilsmeier reagent. Then compound 3c (19.59 g, $0.072 \mathrm{~mol})$ in DMF $(20 \mathrm{ml})$ was added drop wise into the Vilsmeier 
Table 1. Spectral data of the newly prepared compounds 3-31.

Compd. No. Spectral Data

FT-IR (KBr $\left.v_{\max } \mathrm{cm}^{-1}\right): 3436(\mathrm{NH}), 3042,2911,2856(\mathrm{CH}), 1610(\mathrm{C}=\mathrm{N})$.

1H NMR (DMSO-d6) $\delta$ ppm: 2.50 (s, 3H, $\mathrm{CH}_{3}$ ), 6.82 (s, 1H, H-3 indole), 6.97 - 7.07 (m, 4H. Ar-H), 7.25 (d, 1H, indole proton), 7.36 - $7.73(\mathrm{~m}, 2 \mathrm{H}$, indole proton), $7.76(\mathrm{~d}, 1 \mathrm{H}$, indole proton), $11.45(\mathrm{~s}, 1 \mathrm{H}, \mathrm{NH})$. MS. $m / z$ (\%): 207 (M+1 100), 192 (3.12), 180 (3.18), 116 (1.99), 89 (20.56), 69 (29.66).

FT-IR (KBr $\left.v_{\max } \mathrm{cm}^{-1}\right)$ : 3366, 3260, $3180\left(\mathrm{NH}_{2}, \mathrm{NH}\right), 2915,2855(\mathrm{CH}), 1599(\mathrm{C}=\mathrm{N})$.

1H NMR (DMSO-d6) $\delta$ ppm: $6.54\left(\mathrm{~s}, 2 \mathrm{H}, \mathrm{NH}_{2}\right), 6.70-7.21(\mathrm{~m}, 5 \mathrm{H}, \mathrm{Ar}-\mathrm{H}$ and $\mathrm{H}-3$
$7.71-7.82$ (m, 2H, indole proton), $8.19(\mathrm{~d}, 1 \mathrm{H}$, indole proton), $9.13(\mathrm{~s}, 1 \mathrm{H}, \mathrm{NH})$.

FT-IR (KBr $\left.v_{\max }, \mathrm{cm}^{-1}\right)$ : $3182(\mathrm{NH}), 3057,2977,2867$ (CH). indole proton), $12.43(\mathrm{~s}, 1 \mathrm{H}, \mathrm{NH})$.

FT-IR (KBr $\left.v_{\max } \mathrm{cm}^{-1}\right)$ : 3426 (NH), 3037, 2917, $2851(\mathrm{CH}), 1671(\mathrm{C}=\mathrm{O})$

1H NMR (DMSO-d6) $\delta$ ppm: 5.46 (s, 1H, H-3 indole proton), 7.14 - 7.95 (m, 9H, Ar-H and indole proton), 8.20 (s, 1H, $\mathrm{NH})$.

MS. $m / z$ (\%): 261 ( $\left.\mathrm{M}^{+}, 18.48\right), 193$ (2.80), 145 (18.01), 116 (13.51), 69 (100).

FT-IR (KBr, $\left.v_{\max } \mathrm{cm}^{-1}\right)$ : $3181(\mathrm{NH})$; 3063, 2978, 2868, $2713(\mathrm{CH}), 1672(\mathrm{C}=\mathrm{O}), 1578(\mathrm{C}=\mathrm{N})$.

1H NMR (DMSO-d6) $\delta$ ppm: 7.22 - 7.32 (m, 4H, Ar-H), 7.50 (d, 1H, indole proton), $7.72-7.82$ (m, 2H, indole proton), $8.19\left(\mathrm{~d}, 1 \mathrm{H}\right.$, indole proton), $9.95(\mathrm{~s}, 1 \mathrm{H}, \mathrm{CHO}), 12.40\left(\mathrm{~s}, 1 \mathrm{H}, \mathrm{NH}\right.$ exchanged by $\left.\mathrm{D}_{2} \mathrm{O}\right)$.

MS. m/z (\%): 300 (M+1 100, \%), 220 (47), 219 (90.8), 190 (53), 165 (19.1), 143 (15.7).

FT-IR (KBr, $\left.v_{\max } \mathrm{cm}^{-1}\right)$ : 3421, 3385, $3129\left(\mathrm{NH}_{2}, \mathrm{NH}\right), 3048,2965,2860(\mathrm{CH}), 1607(\mathrm{C}=\mathrm{N}), 1578(\mathrm{C}=\mathrm{C})$. $8.42\left(\mathrm{~d}, 1 \mathrm{H}\right.$, indole proton), $8.91(\mathrm{~s}, 1 \mathrm{H},=\mathrm{CH}), 12.06\left(\mathrm{~s}, 1 \mathrm{H}, \mathrm{NH}\right.$ exchanged by $\left.\mathrm{D}_{2} \mathrm{O}\right), 4.34\left(\mathrm{~s}, 2 \mathrm{H}, \mathrm{NH}_{2}\right.$ exchanged by $\left.\mathrm{D}_{2} \mathrm{O}\right)$.

FT-IR (KBr, $\left.v_{\max } \mathrm{cm}^{-1}\right)$ : 3249 (NH), 3056, 2921, $2855(\mathrm{CH}), 1592(\mathrm{C}=\mathrm{N}), 1532(\mathrm{C}=\mathrm{C})$.

$1 \mathrm{H}$ NMR (DMSO-d6) $\delta$ ppm: $7.04-8.10(\mathrm{~m}, 13 \mathrm{H}, \mathrm{Ar}-\mathrm{H}), 6.75(\mathrm{~s}, 1 \mathrm{H},=\mathrm{CH}), 8.22\left(\mathrm{~s}, 1 \mathrm{H}, \mathrm{NH}\right.$ exchanged by $\left.\mathrm{D}_{2} \mathrm{O}\right), 4.33(\mathrm{~s}$, $1 \mathrm{H}$, NH exchanged by $\left.\mathrm{D}_{2} \mathrm{O}\right)$.

MS. $m / z$ (\%): 391( $\left.\mathrm{M}^{+}+1,26.6\right), 389\left(\mathrm{M}^{+}\right.$- 1, 30.5), 233 (2.1), 298 (100), 284 (12.3), 271 (46.9), 190 (60.5).

FT-IR (KBr, $\left.v_{\max } \mathrm{cm}^{-1}\right)$ : $3181(\mathrm{NH}), 3050,2921(\mathrm{CH}), 1600(\mathrm{C}=\mathrm{N}), 1573(\mathrm{C}=\mathrm{C})$.

1H NMR (DMSO-d6) $\delta$ ppm: 7.06 - 7.24 (m, 4H, Ar-H), 7.26 - 7.31 (m, 2H, benothiazole proton), 7.38 (d, 1H, benothiazole proton), 7.47 (d, 1H, indole proton),7.61-7.64 (m, 2H, indole proton), 7.73 (d, 1H, benothiazole proton), 8.36 (d, $1 \mathrm{H}$, indole proton), $8.42(\mathrm{~s}, 1 \mathrm{H},=\mathrm{CH}), 7.95\left(\mathrm{~s}, 1 \mathrm{H}, \mathrm{NH}\right.$ exchanged by $\left.\mathrm{D}_{2} \mathrm{O}\right), 11.82\left(\mathrm{~s}, 1 \mathrm{H}, \mathrm{NH}\right.$ exchanged by $\left.\mathrm{D}_{2} \mathrm{O}\right)$. MS. m/z (\%): 447 (M+11.24), 313 (13.70), 296 (10.33), 204 (15.63), 150 (100).

FT-IR (KBr, $\left.v_{\max } \mathrm{cm}^{-1}\right)$ : 3207(NH), 3054, 3008, 2932, $2862(\mathrm{CH}), 1652(\mathrm{C}=\mathrm{O}), 1611(\mathrm{C}=\mathrm{N}), 1573(\mathrm{C}=\mathrm{C})$. 1H NMR (DMSO-d6) $\delta$ ppm: $7.14-8.80$ (m, 14H, -Ar-H), 4.89 (s, 2H, $\left.\mathrm{CH}_{2}\right), 8.90$ (s, 1H, =CH), $11.92(\mathrm{~s}, 1 \mathrm{H}, \mathrm{NH}$ exchanged by $\left.\mathrm{D}_{2} \mathrm{O}\right), 12.05$ (s, $1 \mathrm{H}$, $\mathrm{NH}$ exchanged by $\left.\mathrm{D}_{2} \mathrm{O}\right)$.

FT-IR (KBr, $\left.v_{\max } \mathrm{cm}^{-1}\right)$ : 3389, $3165(\mathrm{NH}), 3054,2962,2848(\mathrm{CH}), 1654(\mathrm{C}=\mathrm{O}), 1604(\mathrm{C}=\mathrm{N})$.

1H NMR (DMSO-d6) $\delta$ ppm: 3.78 (s, 2H,CH $), 7.16$ - 7.28 (m, 5H, Ar-H and H-2 indole), 7.45 (d, 2H, indole proton), 7.56 - 7.89 (m, 4H, indole proton), 8.41 (d, 2H, indole proton), 8.90 (s, 1H, =CH), 4.31 (s, 1H, NH exchanged by $\mathrm{D}_{2} \mathrm{O}$ ), 12.03 (s, $2 \mathrm{H}, \mathrm{NH}$ exchanged by $\left.\mathrm{D}_{2} \mathrm{O}\right)$.

MS. $m / z$ (\%): 471 (M+1, 0.94), 211 (4.96), 203 (74.04), 177 (12.11), 159 (13.50), 136 (37.99), 91 (100).

FT-IR (KBr, $\left.v_{\max } \mathrm{Cm}^{-1}\right)$ : 3419, 3379, $3273(\mathrm{NH}), 3051,2918,2856(\mathrm{CH}), 1658(\mathrm{C}=\mathrm{O}), 1604(\mathrm{C}=\mathrm{N})$.

1H NMR (DMSO-d6) $\delta$ ppm: 1.95 - 2.03 (m, 2H, CH CH $_{2}, 2.20$ (t, 2H,CH $), 2.71\left(\mathrm{t}, 2 \mathrm{H}, \mathrm{CH}_{2}\right), 6.93-7.33$ (m, 5H, Ar- $\mathrm{H}$ and $\mathrm{H}-2$ indole), 7.42 (d, $2 \mathrm{H}$, indole proton), $7.49-7.83$ (m, 4H, indole proton), 8.12 (d,1H, indole proton), 8.35 (d, $1 \mathrm{H}$, indole proton), $8.27(\mathrm{~s}, 1 \mathrm{H},=\mathrm{CH}), 10.75\left(\mathrm{~s}, 1 \mathrm{H}, \mathrm{NH}\right.$ exchanged by $\left.\mathrm{D}_{2} \mathrm{O}\right), 10.99\left(\mathrm{~s}, 1 \mathrm{H}, \mathrm{NH}\right.$ exchanged by $\left.\mathrm{D}_{2} \mathrm{O}\right), 11.85(\mathrm{~s}, 1 \mathrm{H}, \mathrm{NH}$ exchanged by $\left.\mathrm{D}_{2} \mathrm{O}\right)$.

FT-IR (KBr, $v_{\max } \mathrm{cm}^{-1}$ ): 3426, $3168(\mathrm{NH}), 3098,3048,2953,2849$ (CH), 1648 (C=O), 1608 (C=N).

1H NMR (DMSO-d6) $\delta$ ppm: 7.17 - 7.29 (m, 5H, Ar-H and H-3 benzofuran), 7.46 (d, 2H, indol and benzofuran),7.65 $7.85(\mathrm{~m}, 4 \mathrm{H}$, indole and benzofuran), 8.42 (d, 2H, indole and benzofuran), $8.90(\mathrm{~s}, 1 \mathrm{H},=\mathrm{CH}), 7.95(\mathrm{~s}, 1 \mathrm{H}, \mathrm{NH}$ exchanged by $\left.\mathrm{D}_{2} \mathrm{O}\right), 12.04$ (s, $1 \mathrm{H}$, $\mathrm{NH}$ exchanged by $\left.\mathrm{D}_{2} \mathrm{O}\right)$.

MS. m/z (\%): 458 (M+1, 0.10), 313 (0.11), 296 (5.76), 52 (66.65), 204 (63.53), 133 (43.88), 91 (100).

FT-IR (KBr, $\left.v_{\max } \mathrm{cm}^{-1}\right)$ : 3394, $3171(\mathrm{NH}), 3045,2961,2918(\mathrm{CH}), 1650(\mathrm{C}=\mathrm{O}), 1604(\mathrm{C}=\mathrm{N}), 2205$ (CN). $1 \mathrm{H}$ NMR (DMSO-d6) $\delta$ ppm: 4.33 (s, 2H, $\mathrm{CH}_{2}$ ), 7.16 - 7.28 (m,4H, Ar-H),7.44 (d, 1H, indole proton), 7.55 - 7.79 (m, 2H, indole proton), $8.11\left(\mathrm{~d}, 1 \mathrm{H}\right.$, indole proton), $8.33(\mathrm{~s}, 1 \mathrm{H}, \mathrm{N}=\mathrm{CH}), 11.33\left(\mathrm{~s}, 1 \mathrm{H}, \mathrm{NH}\right.$ exchanged by $\left.\mathrm{D}_{2} \mathrm{O}\right), 11.93(\mathrm{~s}, 1 \mathrm{H}, \mathrm{NH}$ exchanged by $\left.\mathrm{D}_{2} \mathrm{O}\right)$.

${ }^{13}$ C NMR (DMSO-d6) $\delta$ ppm: $24.39\left(\mathrm{CH}_{2}\right), 116.19(\mathrm{CN}), 157.73$ (C=N), 163.58 (CO), 107.75, 111.44, 111.66, 120.95, 122.14, 122.27, 123.13, 125.09, 130.07, 130.97, 131.16, 131.73, 131.89, 136.46. 


\section{Continued} exchanged by $\mathrm{D}_{2} \mathrm{O}$ ), 11.91 ( $\mathrm{s}, 1 \mathrm{H}, \mathrm{NH}$ exchanged by $\mathrm{D}_{2} \mathrm{O}$ ).

${ }^{13} \mathrm{C}$ NMR (DMSO-d6) $\delta$ pm: $10.65\left(\mathrm{CH}_{3}\right), 116.19(\mathrm{CN}), 155.22(\mathrm{C}=\mathrm{N}), 159(\mathrm{C}=\mathrm{NNH}), 111.41,119.24,122.18,129.79$, $130.07,130.85,131.04,131.15,131.24,131.79,133.31,136.46,136.46,136.76$.

FT-IR (KBr, $v_{\max } \mathrm{cm}^{-1}$ ): 3335, 3126(NH), 3055, $2921(\mathrm{CH}), 1686(\mathrm{C}=\mathrm{O}), 2204(\mathrm{CN})$. 11.73 (s, $1 \mathrm{H}, \mathrm{NH}$ exchanged by $\left.\mathrm{D}_{2} \mathrm{O}\right), 12.04$ (s, $1 \mathrm{H}$, $\mathrm{NH}$ exchanged by $\mathrm{D}_{2} \mathrm{O}$ ).

MS, m/z (\%), 611 (M+ 0.63), 392 (1.05), 379 (0.51), 358 (11.477), 327 (1.42), 217 (33.11), 77.02 (100).

FT-IR (KBr, $v_{\max } \mathrm{cm}^{-1}$ ): 3233, 3166 (NH), 3091, $2971(\mathrm{CH}), 1682(\mathrm{C}=\mathrm{O}), 2208(\mathrm{CN})$.

1H NMR (DMSO-d6) $\delta$ ppm: $7.22-8.39$ (m, 12H, Ar-H), 8.68 (s, 1H, =CH), 8.88 (s, 1H, N=CH), 11.93 (s, $1 \mathrm{H}, \mathrm{NH}$ exchanged by $\left.\mathrm{D}_{2} \mathrm{O}\right), 12.42$ (s, $1 \mathrm{H}, \mathrm{NH}$ exchanged by $\left.\mathrm{D}_{2} \mathrm{O}\right)$.

FT-IR (KBr, $\left.v_{\max } \mathrm{cm}^{-1}\right)$ : 3372, $3155(\mathrm{NH}), 3093,2930,2853(\mathrm{CH}), 1712(\mathrm{C}=\mathrm{O})$.

$1 \mathrm{H}$ NMR (DMSO-d6) $\delta$ ppm: 5.17 (s, 1H, H-4 coumarin ring), 6.56 - 8.22 (m, $12 \mathrm{H}, \mathrm{Ar}-\mathrm{H}), 8.72(\mathrm{~s}, 1 \mathrm{H}, \mathrm{N}=\mathrm{CH}), 11.44$ (s, $1 \mathrm{H}, \mathrm{NH}$ exchanged by $\left.\mathrm{D}_{2} \mathrm{O}\right), 11.56$ (s, $1 \mathrm{H}, \mathrm{NH}$ exchanged by $\left.\mathrm{D}_{2} \mathrm{O}\right), 12.14\left(\mathrm{~s}, 1 \mathrm{H}, \mathrm{NH}\right.$ exchanged by $\left.\mathrm{D}_{2} \mathrm{O}\right)$. MS. $m / z$ (\%): 485( $\left.\mathrm{M}^{+}, 0.30\right), 470$ (0.4), 457 (0.4), 442 (0.1), 417 (3.00), 271 (100), 191(61.6), 165 (77.9).

FT-IR (KBr, $\left.v_{\max } \mathrm{cm}^{-1}\right)$ : 3475, 3396, 3369, 3234, $3180\left(2 \mathrm{NH}_{2}, \mathrm{NH}\right), 3059,2934,2838(\mathrm{CH}), 1721,1670$ (2 C=O), 2210 $(\mathrm{CN})$.

1H NMR (DMSO-d6) $\delta$ ppm: 1.26 (t, 3H, $\mathrm{CH}_{2}-\underline{\mathrm{CH}_{3}}$ ), 4.23 (q, 2H, $\underline{\mathrm{CH}_{2}}-\mathrm{CH}_{3}$ ), $7.22-8.225$ (m, $12 \mathrm{H}, \mathrm{Ar}-\mathrm{H}$ and 2 NH ), $8.25(\mathrm{~s}, 1 \mathrm{H}, \mathrm{N}=\mathrm{CH}), 12.91\left(\mathrm{~s}, 1 \mathrm{H}, \mathrm{NH}\right.$ exchanged by $\left.\mathrm{D}_{2} \mathrm{O}\right), 12.45\left(\mathrm{~s}, 1 \mathrm{H}, \mathrm{NH}\right.$ exchanged by $\left.\mathrm{D}_{2} \mathrm{O}\right)$.

FT-IR (KBr, $\left.v_{\max } \mathrm{cm}^{-1}\right)$ : 3395, 3269, $3164\left(\mathrm{NH}_{2}, \mathrm{NH}\right), 3049,2974,2866(\mathrm{CH}), 1665(\mathrm{C}=\mathrm{O}), 1237$ (C=S).

$1 \mathrm{H}$ NMR (DMSO-d6) $\delta$ ppm: $7.16-8.48$ (m, $15 \mathrm{H}, \mathrm{Ar}-\mathrm{H}$ and $\mathrm{NH}_{2}$ ), 8.90(s, $\left.1 \mathrm{H}, \mathrm{N}=\mathrm{CH}\right), 11.00$ (s, $1 \mathrm{H}$, NH exchanged by $\mathrm{D}_{2} \mathrm{O}$ ), 12.43 (s, $1 \mathrm{H}, \mathrm{NH}$ exchanged by $\left.\mathrm{D}_{2} \mathrm{O}\right)$.

${ }^{13} \mathrm{C}$ NMR (DMSO-d6) $\delta$ ppm: $147.39(\mathrm{C}=\mathrm{N}), 153\left(\mathrm{C}-\mathrm{NH}_{2}\right), 161.36$ (CO), 183.91 (CS), 112.10, 113.61, 121.01, 122.38, 123.44, 125.7, 126.93, 130.7, 131.14, 131.87, 135.88, 136.51, 136.51, 141.72.

FT-IR (KBr, $\left.v_{\max } \mathrm{cm}^{-1}\right)$ : $3129(\mathrm{NH}), 3091,3052,2947,2860(\mathrm{CH}), 1681(\mathrm{C}=\mathrm{O}), 1239(\mathrm{C}=\mathrm{S})$. $1 \mathrm{H}$ NMR (DMSO-d6) $\delta$ ppm: 2.25 (s, 3H, $\mathrm{CH}_{3}$ ), 7.05-7.81(m, $\left.13 \mathrm{H}, \mathrm{Ar}-\mathrm{H}\right), 8.22(\mathrm{~s}, 1 \mathrm{H}, \mathrm{CH}), 12.40$ (s, $1 \mathrm{H}, \mathrm{NH}$ exchanged by $\left.\mathrm{D}_{2} \mathrm{O}\right)$.

FT-IR (KBr, $\left.v_{\max } \mathrm{cm}^{-1}\right): 3269(\mathrm{NH}), 3090,2970,2869(\mathrm{CH})$.

1H NMR (DMSO-d6) $\delta$ ppm: 6.85 (s, 1H, N=CH), 6.98-7.32 (m, 10 H, Ar-H and H-5 thiazole ring), 7.50 (d, $1 \mathrm{H}$, indole proton), 7.65 - 7.82 (m, 2H, indole proton), 8.20 - 8.22 (d, 1H, indole proton), 12.43 (s, 1H, NH exchanged by $\mathrm{D}_{2} \mathrm{O}$ ).

FT-IR (KBr, $\left.v_{\max } \mathrm{cm}^{-1}\right)$ : $3127(\mathrm{NH}), 3084,2967,2862(\mathrm{CH}), 1671,1718(2 \mathrm{C}=\mathrm{O})$.

1H NMR (DMSO-d6) $\delta$ ppm: 7.22 - 7.32 (m, 5 H, Ar-H and H-4 chromene), 7.44 (d, 2H, indole and chromene), 7.70 7.92 (m, 4H, indole and chromene), 7.93 - 7.97 (m, 2H, CH= CH), 8.00 - 8.22 (d, 2H, indole and chromene), 12.43 (s, $1 \mathrm{H}$, $\mathrm{NH}$ exchanged by $\left.\mathrm{D}_{2} \mathrm{O}\right)$.

FT-IR (KBr, $\left.v_{\max } \mathrm{cm}^{-1}\right)$ : 3466, 3310, $3149\left(\mathrm{NH}_{2}, \mathrm{NH}\right), 3048,2970,2865(\mathrm{CH}), 1688(\mathrm{C}=\mathrm{O})$ and $2203(\mathrm{CN})$. 1H NMR (DMSO-d6) $\delta$ ppm: 7.22 - 7.32 (m, $6 \mathrm{H}, \mathrm{Ar}-\mathrm{H}$ and $\mathrm{NH}_{2}$ proton), $7.47-7.83$ (m, 3H, indole proton), 7.96 (d, $1 \mathrm{H}$, indole proton), 8.22 (s, $1 \mathrm{H}, \mathrm{C}=\mathrm{CH}), 12.43$ (s, $1 \mathrm{H}, \mathrm{NH}$ exchanged by $\left.\mathrm{D}_{2} \mathrm{O}\right)$.

FT-IR (KBr, $v_{\max } \mathrm{cm}^{-1}$ ): $3269(\mathrm{NH}), 3047,2973,2861(\mathrm{CH}), 2208(\mathrm{CN}), 1708(\mathrm{C}=\mathrm{O})$.

1H NMR (DMSO-d6) $\delta$ ppm: 1.03 (t, 3H, $\mathrm{CH}_{2} \mathrm{CH}_{3}$ ), 4.23 (q, 2H, $\underline{\mathrm{CH}_{2}} \mathrm{CH}_{3}$ ), 7.29 - 7.35 (m, 4H, Ar-H), 7.56 - 7.86 (m, $3 \mathrm{H}$, indole proton), $8.15-8.17$ (d, $1 \mathrm{H}$, indole proton), 8.25 (s, $1 \mathrm{H},=\mathrm{CH}) ; 4.34\left(\mathrm{~s}, 1 \mathrm{H}, \mathrm{NH}\right.$ exchanged by $\left.\mathrm{D}_{2} \mathrm{O}\right)$. MS. $m / z$ (\%): 395 (M+11.8), 321 (44.6), 242 (100), 215 (17.6), 214 (32.8).

FT-IR (KBr, $\left.v_{\max } \mathrm{cm}^{-1}\right)$ : $3303(\mathrm{NH}), 2203(\mathrm{CN}), 3052,2961,2921,2859(\mathrm{CH}), 1672(\mathrm{C}=\mathrm{O})$. $3 \mathrm{H}$, indole proton), 7.97 (d, IH, indole proton), 10.18 (s, $1 \mathrm{H}, \mathrm{NH}$ exchanged by $\left.\mathrm{D}_{2} \mathrm{O}\right), 11.41$ (s, $1 \mathrm{H}, \mathrm{NH}$ exchanged by $\mathrm{D}_{2} \mathrm{O}$ ), 12.22 (s, $1 \mathrm{H}, \mathrm{NH}$ exchanged by $\mathrm{D}_{2} \mathrm{O}$ ).

FT-IR (KBr, $\left.v_{\max } \mathrm{cm}^{-1}\right)$ : $3384(\mathrm{NH}), 2212(\mathrm{CN}), 3054,2957,2852(\mathrm{CH})$.

1H NMR (DMSO-d6) $\delta$ ppm: 7.21 - 7.32 (m, 4H, Ar-H), 7.57 - 7.95 (m, 8H, indole and benzimidazole proton), 8.31 (s, $1 \mathrm{H},=\mathrm{CH}), 12.57\left(\mathrm{~s}, 1 \mathrm{H}, \mathrm{NH}\right.$ exchanged by $\left.\mathrm{D}_{2} \mathrm{O}\right), 12.88\left(\mathrm{~s}, 1 \mathrm{H}, \mathrm{NH}\right.$ exchanged by $\left.\mathrm{D}_{2} \mathrm{O}\right)$.

FT-IR (KBr, $v_{\max } \mathrm{cm}^{-1}$ ): $3355(\mathrm{OH}), 3179(\mathrm{NH})$, 1623, 1707 (2C=O), 3095, 2974, $2865(\mathrm{CH}), 1242$ (C=S).

1H NMR (DMSO-d6) $\delta$ ppm: 4.71 (s, 2H, $\mathrm{CH}_{2}$ ), 7.32 - 7.33 (m, 4H, Ar-H), 7.50 (d, 1H, indole proton), 7.66 - 7.79 (m, 


\section{Continued}

26

FT-IR (KBr, $\left.v_{\max } \mathrm{cm}^{-1}\right)$ : 3249, 3154 (2 NH), 3026, 2814 (CH), 1689 (C=O), 1283 (C=S).

$1 \mathrm{H}$ NMR (DMSO-d6) $\delta$ ppm: 3.21 (s, 2H, $\mathrm{CH}_{2}$ ), 6.79 - 7.79 (m, 6H, Ar-H and $\mathrm{H}-5$ thiazole), 8.31 (s, $1 \mathrm{H}, \mathrm{NH}$ exchanged by $\left.\mathrm{D}_{2} \mathrm{O}\right), 9.34$ (s, $1 \mathrm{H}, \mathrm{NH}$ exchanged by $\left.\mathrm{D}_{2} \mathrm{O}\right)$.

FT-IR (KBr, $\left.v_{\max } \mathrm{cm}^{-1}\right)$ : 3232, 3260, $3176(\mathrm{NH}), 1668(\mathrm{C}=\mathrm{O}), 3053,2990,2873(\mathrm{CH}), 1242(\mathrm{C}=\mathrm{S})$.

1H NMR (DMSO-d6) $\delta$ ppm: 6.95 - 7.31 (m, 10 H, Ar-H and H-5 thiazole), 7.50 - 7.90 (m, 4H, indole proton), 9.96 (s,

$1 \mathrm{H},=\mathrm{CH}), 12.18\left(\mathrm{~s}, 1 \mathrm{H}, \mathrm{NH}\right.$ exchanged by $\left.\mathrm{D}_{2} \mathrm{O}\right), 12.22\left(\mathrm{~s}, 1 \mathrm{H}, \mathrm{NH}\right.$ exchanged by $\left.\mathrm{D}_{2} \mathrm{O}\right), 12.40(\mathrm{~s}, 1 \mathrm{H}, \mathrm{NH}$ exchanged by $\left.\mathrm{D}_{2} \mathrm{O}\right)$.

${ }^{13}$ C NMR (DMSO-d6) $\delta$ ppm: 185.37 (CS), 168 (CO), 155.46 (C=C), 166 (C-NH), 108.01, 112.01, 112.01, 113.74, 121.01, 123.44, 125.70, 127.62, 128.93, 129.01, 130.21, 130.8, 134.46, 135.88, 144.51, 146.01, 148.01 .

FT-IR (KBr, $\left.v_{\max } \mathrm{cm}^{-1}\right): 3275(\mathrm{NH}), 1706(\mathrm{C}=\mathrm{O}), 3037,2988,2817(\mathrm{CH})$.

1H NMR (DMSO-d6) $\delta$ ppm: 2.38 (s, 3H, $\left.\mathrm{CH}_{3}\right), 7.31-7.35(\mathrm{~m}, 9 \mathrm{H}, \mathrm{Ar}-\mathrm{H}), 7.36-7.53(\mathrm{~m}, 4 \mathrm{H}$, indole proton and =CH),

$7.82\left(\mathrm{~d}, 1 \mathrm{H}\right.$, indole proton), $12.40\left(\mathrm{~s}, 1 \mathrm{H}, \mathrm{NH}\right.$ exchanged by $\left.\mathrm{D}_{2} \mathrm{O}\right)$.

${ }^{13} \mathrm{C}$ NMR (DMSO-d6) $\delta$ ppm: $12.58\left(\mathrm{CH}_{3}\right), 153(\mathrm{C}=\mathrm{C}), 160(\mathrm{C}=\mathrm{O}), 108.82,120.62,120.74,126.43,126.63,128.87$, 129.07, 137.15, 140.16, 140.34 .

MS. $m / z$ (\%): 456 (M+1 1.9), 441 (0.3), 413 (0.4), 336 (3.4), 359 (48.1), 358 (100), 341 (62.3), 266 (20.3), 77 (92.7).

29a

FT-IR (KBr, $\left.v_{\max }, \mathrm{cm}^{-1}\right)$ : 3428, $3198(\mathrm{NH}), 3039,2865(\mathrm{CH})$.

1H NMR (DMSO-d6) $\delta$ ppm: 2.26 (s, 3H, $\mathrm{CH}_{3}$ ), 6.93 - 7.18 (m, 9H, Ar-H), 7.29 - 7.82 (m, 4H, indole proton), 8.04 (s, 1H, $\mathrm{NH}$ exchanged by $\left.\mathrm{D}_{2} \mathrm{O}\right), 11.56$ (s, $1 \mathrm{H}$, NH exchanged by $\left.\mathrm{D}_{2} \mathrm{O}\right)$.

$29 b$

FT-IR (KBr, $\left.v_{\max } \mathrm{cm}^{-1}\right)$ : 3209 (NH), 3048, 2917, $2863(\mathrm{CH})$.

1H NMR (DMSO-d6) $\delta$ ppm: 2.21 (s, 3H, $\mathrm{CH}_{3}$ ), 6.93 - 7.32 (m, 14H, Ar-H), 7.37 - 7.82 (m, 4H, indole proton),

$11.56\left(\mathrm{~s}, 1 \mathrm{H}, \mathrm{NH}\right.$ exchanged by $\left.\mathrm{D}_{2} \mathrm{O}\right)$.

MS. m/z (\%): 544 (M+3.3), 529 (2.3), 467 (22.9), 375 (15.41), 295 (6.2), 298 (100), 273 (71.8), 271 (17.2).

30

FT-IR (KBr, $v_{\max } \mathrm{cm}^{-1}$ ): 3323, 3174 (NH), 3055, 2969, 2864 (CH), 1242 (C= S).

1H NMR (DMSO-d6) $\delta$ ppm: 2.24 (s, 3H, $\mathrm{CH}_{3}$ ), 2.28 (s, 3H, $\mathrm{CH}_{3}$ ), 7.11 - 7.35 (m, 13H, Ar-H), 7.37 - 7.86 (m, 4H, indole proton), 11.48 (s, $1 \mathrm{H}, \mathrm{NH}$ exchanged by $\mathrm{D}_{2} \mathrm{O}$ ), 12.00 (s, $1 \mathrm{H}, \mathrm{NH}$ exchanged by $\mathrm{D}_{2} \mathrm{O}$ ). MS. m/z (\%): 617 (M+ $\left.\mathrm{M}^{+}, 0.46\right), 587$ (0.54), 522 (0.46), 467 (0.63), 414 (1.35), 330 (2.73), 252 (57.01), 125 (100).

reagent and continuous stirring and kept at room temperature for $2 \mathrm{~h}$. The reaction mixture was allowed to stand overnight and was then refluxed for $2 \mathrm{~h}$. under vigorous stirring. The mixture was then poured onto ice cold water and neutralized with dilute ammonia solution till the precipitation occurs. The formed precipitate was collected by filtration and recrystallized from ethanol to give 4 as yellow crystals. Yield (15.13 g, 70\%, m.p.: 270-272 ${ }^{\circ}$ C. Anal. calcd. for $\mathrm{C}_{15} \mathrm{H}_{10} \mathrm{Br} \mathrm{NO}$ (300.15): C, 60.02; H, 3.36; Br, 26.62; N, 4.67. Found: C, 59.89; $\mathrm{H}$, 3.16; Br, 26.42; N, 4.37.

\subsubsection{General Procedure for the Synthesis of 5a-c}

An equimolecular mixture of 4 ( $3 \mathrm{~g}, 0.01 \mathrm{~mol})$ and the hydrazine derivatives $(0.5 \mathrm{ml}, 0.01 \mathrm{~mol})$ were refluxed in absolute ethanol $(20 \mathrm{ml})$ in the presence of 2 - 3 drops of glacial acetic acid for the appropriate time. The reaction mixture was cooled to room temperature and poured into ice-cold water. The separated product was filtered, washed with cold water, dried and recrystallized from the appropriate solvent to give 5a-c.

\section{1-[2-(4-Bromophenyl)-1H-indol-3-ylmethylene]hydrazine 5a}

Compound 5a was prepared from hydrazine hydrate for $1 \mathrm{~h}$. Orange crystals. Yield (2.48 g, 79\%); m.p.: 338-340 ${ }^{\circ} \mathrm{C}$ (xylene). Anal. calcd. for $\mathrm{C}_{15} \mathrm{H}_{12} \mathrm{Br} \mathrm{N}_{3}$ (314.18): C, 57.34; H, 3.85; Br, 25.43; N, 13.37. Found: C, 57.24; H, 3.65; Br, 25.33; N, 13.17.

\section{1-[ 2-(4-Bromophenyl)-1H-indol-3-ylmethylene]-2-phenyl-hydrazine 5b}

Compound $\mathbf{5 b}$ was prepared from phenyl hydrazine for 4 h. Pale brown powder. Yield (2.5 g, 64\%); m.p.: $115-117^{\circ} \mathrm{C}$ (hexane). Anal.calcd. for $\mathrm{C}_{21} \mathrm{H}_{16} \mathrm{BrN}_{3}$ (390.28): C, 64.63; H, 4.13; Br, 20.47; N, 10.77. Found: C, 64.43; H, 4.00; Br, 20.27; N, 10.57.

\section{2-\{2-[2-(4-Bromophenyl)-1H-indol-3-ylmethylene]hydrazine\}-1,3-benzothiazole 5c}

Compound 5c was prepared from 2-hydrazinyl-1,3-benzothiazole for $4 \mathrm{~h}$. Pale yellow crystal. Yield (2.46 g, 55\%); m.p.: 280-282 ${ }^{\circ} \mathrm{C}$ (ethanol/DMF). Anal. calcd. for $\mathrm{C}_{22} \mathrm{H}_{15} \mathrm{BrN}_{4} \mathrm{~S}$ (447.35): C, 59.07; H, 3.38; Br, 17.86; N, 12.52; S, 7.17. Found: C, 58.98; H, 3.18; Br, 17.66; N, 12.40; S, 7.00. 


\subsubsection{General Procedure for the Synthesis of 7-10}

An equimolecular mixture of 4 ( 3 g,0.01 mol) and the acid hydrazide derivatives $6 \mathbf{6 a - d}(0.01 \mathrm{~mol})$ was refluxed for $2 \mathrm{~h}$. in absolute ethanol $(20 \mathrm{ml})$ in the presence of 2 - 3 drops of glacial acetic acid. The reaction mixture was cooled to room temperature and poured into ice-cold water. The separated product was filtered, washed with cold water, dried and recrystallized from the appropriate solvent.

$N^{\prime}$-[2-(4-Bromophenyl)-1 $\mathrm{H}$-indol-3-ylmethylene]-2-(quinolin-8-yloxy)acetohydrazide 7

Yellow crystals. Yield (3 g, 60\%); m.p.: 290-292 ${ }^{\circ} \mathrm{C}$ (ethanol/DMF). Anal. calcd for $\mathrm{C}_{26} \mathrm{H}_{19} \mathrm{BrN}_{4} \mathrm{O}_{2}$ (499.35):

C, 62.54; H, 3.84; Br, 16.00; N, 11.22. Found: C, 62.24; H, 3.62; Br, 15.88; N, 11.02 .

$N^{\prime}$-(2-(4-Bromophenyl)-1H-indol-3-ylmethylene)-2-(1H-indol-3-yl)acetohydrazide 8

Red crystals. Yield (2.83 g, 60\%); m.p.: $330-332^{\circ} \mathrm{C}$ (ethanol). Anal. calcd for $\mathrm{C}_{25} \mathrm{H}_{19} \mathrm{BrN}_{4} \mathrm{O}$ (471.35): C, 63.70; H, 4.06; Br, 16.95; N, 11.89. Found: C, 63.40; H, 4.00; Br, 16.65; N, 11.59.

$N^{\prime}$-[2-(4-Bromophenyl)-1H-indol-3-ylmethylene]-4-(1H-indol-3-yl)-butanehydrazide 9

Dark yellow powder. Yield (3.75 g, 75\%); m.p.: $170-172^{\circ} \mathrm{C}$ (xylene). Anal.calcd for $\mathrm{C}_{27} \mathrm{H}_{23} \mathrm{BrN}_{4} \mathrm{O}$ (499.40):

C, 64.94; H, 4.64; Br, 16.00; N, 11.22. Found: C, 64.74; H, 4.34; Br, 15.88; N, 11.00 .

$N^{\prime}$-[ 2-(4-Bromophenyl)-1H-indol-3-ylmethylene]-benzofuran-2-carbohydrazide 10

Yellow crystals. Yield (2.75 g, 60\%); m.p.: $320-322^{\circ} \mathrm{C}$ (ethanol/DMF). Anal. calcd for $\mathrm{C}_{24} \mathrm{H}_{16} \mathrm{BrN}_{3} \mathrm{O}_{2}$ (458.30): C, 62.90; H, 3.52; Br, 17.43; N, 9.17. Found: C, 62.70; H, 3.32; Br, 17.23; N, 9.00.

\subsection{5. $N^{\prime}$-[2-(4-Bromophenyl)-1H-indol-3-ylmethylene]-2-cyanoacetohydrazide 11}

An equimolecular mixture of 4 (3 g, $0.01 \mathrm{~mol})$ and cyanoacetohydrazide (1.98 g, $0.02 \mathrm{~mol})$ in absolute ethanol (30 ml) was heated under reflux for $2 \mathrm{~h}$. The precipitate formed after cooling was filtered off, washed with cold ethanol, dried and recrystallized from DMF to give $\mathbf{1 1}$ as pale brown powder. Yield (3.24 g, 85\%); m.p.: 290-292 ${ }^{\circ} \mathrm{C}$. Anal. calcd for $\mathrm{C}_{18} \mathrm{H}_{13} \mathrm{BrN}_{4} \mathrm{O}$ (381.22): C, 56.71; H, 3.44; Br, 20.96; N, 14.70. Found: C, 56.41; H, 3.24; Br, 20.86; N, 14.50 .

\subsection{6. $N^{\prime}$-[2-(4-Bromophenyl)-1H-indol-3-ylmethylene]-2-cyano-2-[(4-methylphenyl)hydrazono]- acetohydrazide 12}

To a cold solution of 11 (3.81 g, $0.01 \mathrm{~mol})$ in ethanol $(20 \mathrm{ml})$ containing sodium acetate $(3.0 \mathrm{~g})$ was added with continuous stirring 4-methylbenzene diazonium salt $(0.01 \mathrm{~mol})$ [prepared by adding sodium nitrite $(1.38 \mathrm{~g}, 0.02$ $\mathrm{mol})$ in water $(8 \mathrm{ml})$ to a cold solution of $p$-toluidine $(1.07 \mathrm{~g}, 0.01 \mathrm{~mol})$ in the appropriate amount of hydrochloric acid]. The reaction mixture was stirred for $2 \mathrm{~h}$. and the formed solid was collected by filtration and recrystallized from ethanol to give 12 as orange crystals. Yield (3.5 g, 70\%); m.p.: 240-242 ${ }^{\circ} \mathrm{C}$. Anal. calcd for $\mathrm{C}_{25} \mathrm{H}_{19}$ Br $\mathrm{N}_{6} \mathrm{O}$ (499.36): C, 60.13; H, 3.84; Br, 16.00; N, 16.83. Found: C, 60.00; H, 3.54; Br, 15.98; N, 16.75.

\subsubsection{General Procedure for the Synthesis of $13 a, b$ and 14}

Equimolecular mixture of $\mathbf{1 1}$ (3.81 g, $0.01 \mathrm{~mol}$ ) and the selected aldehydes such as 1,3-diphenyl- $1 \mathrm{H}$-pyrazole-4carboxaldehyde, $p$-nitrobenzaldehyde and salicyaldehyde $(0.01 \mathrm{~mol})$ in 1,4-dioxane $(20 \mathrm{ml})$ containing piperidine $(0.5 \mathrm{ml})$ was heated under reflux for $3 \mathrm{~h}$. The reaction mixture was left to cool then poured onto ice/water containing few drops of hydrochloric acid and the formed solid product was collected by filtration and recrystallized from the appropriate solvent.

$N^{\prime}$-[2-(4-Bromophenyl-1H-indol-3-ylmethylene]-2-cyano-3-(1,3-diphenyl-1H-pyrazol-4-yl) acrylohydrazide 13a

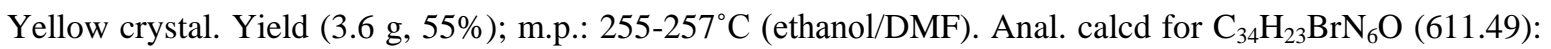
C, 66.78; H, 3.79; Br, 13.07; N, 13.74. Found: C, 66.48; H, 3.59; Br, 13.00; N, 13.55.

$N^{\prime}$-[2-(4-Bromophenyl)-1H-indol-3-ylmethylene]-3-(4-nitrophenyl)-2-cyanoacrylohydrazide 13b

Yellow crystals. Yield (2.31 g, 45\%); m.p.: $230-232^{\circ} \mathrm{C}$ (ethanol). Anal.calcd for $\mathrm{C}_{25} \mathrm{H}_{16} \mathrm{BrN}_{5} \mathrm{O}_{3}$ (514.33): C, 58.38; H, 3.14; Br, 15.54; N, 13.62. Found: C, 58.18; H, 3.00; Br, 15.34; N, 13.32.

$N^{\prime}$-[2-(4-Bromophenyl)-1H-indol-3-ylmethylene]-2-imino-2H-chromene-3-carbohydrazide 14

Brown crystals. Yield (2.9 g, 60\%); m.p.: $130-132^{\circ} \mathrm{C}$ (hexane). Anal. calcd for $\mathrm{C}_{25} \mathrm{H}_{17} \mathrm{BrN}_{4} \mathrm{O}_{2}$ (485.33): C, 61.87; H, 3.53; Br, 16.46; N, 11.54. Found: C, 61.57; H, 3.33; Br, 16.26; N, 11.34.

\subsubsection{General Procedure for the Synthesis of 15 and 16}

To a solution of compound $\mathbf{1 1}(3.81 \mathrm{~g}, 0.01 \mathrm{~mol})$ in absolute ethanol $(50 \mathrm{ml})$ containing triethylamine $(1 \mathrm{ml})$ either ethyl cyanoacetate $(1.13 \mathrm{~g}, 0.01 \mathrm{~mol})$ or phenylisothiocyanate $(1.39 \mathrm{~g}, 0.01 \mathrm{~mol})$ together with elemental 
sulfur $(0.32 \mathrm{~g}, 0.01 \mathrm{~mol})$ were added. Reaction mixture was heated under reflux for $8 \mathrm{~h}$. then poured onto ice/water mixture and the formed solid product, in each case, was collected by filtration recrystallized from ethanol.

Ethyl 2,4-diamino-5-\{[2-(2-(4-bromophenyl)-1H-indol-3-ylmethylene)hydrazino]-carbonyl\}thiophene-3carboxylate 15

Dark brown crystals. Yield (2.63 g, 50\%); m.p.: $190-192^{\circ} \mathrm{C}$. Anal. calcd for $\mathrm{C}_{23} \mathrm{H}_{20} \mathrm{BrN}_{5} \mathrm{O}_{3} \mathrm{~S}$ (526.40): C, 52.48; H, 3.83; Br,15.18; N, 13.30; S, 6.09. Found: C, 52.28; H, 3.53; Br, 15.00; N, 13.00; S, 6.00.

4-Amino- $N$ '-[2-(4-bromophenyl)-1 $H$-indol-3-ylmethylene]-3-phenyl-2-thioxo-2,3-dihydro-1,3-thiazole-5carbohydrazide 16

Brown crystals. Yield (3.35 g, 61\%); m.p.: 245-247 ${ }^{\circ} \mathrm{C}$. Anal. calcd for $\mathrm{C}_{25} \mathrm{H}_{18} \mathrm{Br} \mathrm{N}_{5} \mathrm{OS}_{2}$ (548.47): C, 54.75; H, 3.31; Br, 14.57; N,12.77; S, 11.69. Found C, 54.55; H, 3.11; Br, 14.37; N, 12.57; S, 11.49.

\subsubsection{6-[(2-(4-Bromophenyl)-1H-indol-3-ylmethylene)amino]-5-methyl-2-thioxo-3-phenyl-2,3-} dihydro-1,3-thiazolo [4,5-d]pyrimidin-7(6H)-one 17

A solution of compound 16 (5.48 g, $0.01 \mathrm{~mol})$ in a mixture of acetic acid (5 ml) and acetic anhydride (10 ml) was heated under reflux for $8 \mathrm{~h}$. and then allowed to cool. The precipitate that formed was collected by filtration, dried and recrystallized from acetic acid to give compound 17 as yellow crystals; Yield (3.4 g, 60\%); m.p.: 316-318 ${ }^{\circ} \mathrm{C}$. Anal. Calcd. $\mathrm{C}_{27} \mathrm{H}_{18} \mathrm{Br} \mathrm{N}_{5} \mathrm{O} \mathrm{S}_{2}$ (572.50): C, 56.64; H, 3.17; Br, 13.96; N, 12.23; S, 11.2. Found: C, 56.44; H, 3.07; Br, 13.66; N, 12.03; S, 11.00.

\subsubsection{0. $N$-[2-(4-Bromophenyl)- $1 H$-indol-3-ylmethylene]-4-phenyl-1,3-thiazol-2-amine 18}

A mixture of compound 4 ( $3 \mathrm{~g}, 0.01 \mathrm{~mol})$, phenacyl bromide $(0.01 \mathrm{~mol})$ and thiourea $(0.78 \mathrm{~g}, 0.01 \mathrm{~mol})$ in absolute ethanol ( $30 \mathrm{ml})$ containing acetic acid $(1 \mathrm{ml})$ were heated under reflux for $8 \mathrm{~h}$. Reaction mixture poured in an ice cold water, the solid obtained was filtered, dried and recrystallized from ethanol to give $\mathbf{1 8}$ as brown powder. Yield (3.21 g, 70\%); m.p.: 230-232 ${ }^{\circ} \mathrm{C}$. Anal. calcd. for $\mathrm{C}_{24} \mathrm{H}_{16} \mathrm{Br} \mathrm{N} \mathrm{N}_{3} \mathrm{~S}$ (458.37): C, 62.89; H, 3.52; $\mathrm{Br}$, 17.43; N, 9.17; S, 7.00. Found: C, 62.59; H, 3.22; Br, 17.23; N, 9.00; S, 6.81.

\subsubsection{3-[3-(2-(4-Bromophenyl)-1H-indol-3-yl)prop-2-enoyl]-2H-chromen-2-on 19}

A mixture of 4 ( $3 \mathrm{~g}, 0.01 \mathrm{~mol})$, 3-acetyl-2H-chromen-2-one (1.88 g, $0.01 \mathrm{~mol})$ in $20 \mathrm{ml}$ absolute ethanol and 0.5 $\mathrm{ml}$ piperdine was refluxed for $30 \mathrm{~min}$. The reaction mixture was left overnight at room temperature, the obtained solid was filtered off and recrystallized from ethanol to give 19 as yellow crystals. Yield (4.01 g, 64\%); m.p.: 280-282 ${ }^{\circ} \mathrm{C}$. Anal. calcd. for $\mathrm{C}_{26} \mathrm{H}_{16} \mathrm{BrNO}_{3}$ (470.31): C, 66.40; H, 3.43; $\mathrm{Br}, 16.99 ; \mathrm{N}, 2.98$. Found: $\mathrm{C}, 66.30 ; \mathrm{H}$, 3.23; $\mathrm{Br}, 16.79 ; \mathrm{N}, 2.78$.

\subsubsection{General Procedure for the Synthesis of $20 \mathrm{a}, \mathrm{b}$ and 22}

To a solution of compound 4 (3 g, $0.01 \mathrm{~mol}$ ) in $20 \mathrm{ml}$ ethanol, the appropriate active methylene compounds such as cyanoacetamide, ethyl cyanoacetate and $1 H$-benzimidazol-2-ylacetonitrile $(0.01 \mathrm{~mol})$ and few drops of triethylamine was added. The reaction mixture was refluxed for $5 \mathrm{~h}$. and then allowed to cool. The formed solid product was collected by filtration, washed with ethanol and recrystallized from the appropriate solvent.

3-[2-(4-Bromophenyl)-1H-indol-3-yl]-2-cyanoprop-2-enamide 20a

Yellow powder. Yield (2.38 g, 65\%); m.p.: $210-212^{\circ} \mathrm{C}$ (ethanol). Anal. calcd for $\mathrm{C}_{18} \mathrm{H}_{12} \mathrm{BrN}_{3} \mathrm{O}$ (366.21): C, 59.03; H, 3.30; Br, 21.82; N, 11.47. Found: C, 58.89; H, 3.00; Br, 21.52; N, 11.17.

Ethyl 3-[2-(4-bromophenyl)-1H-indol-3-yl]-2-cyanoprop-2-enoate 20b

Yellow powder. Yield (2.37 g, 60\%); m.p.: 245-247 ${ }^{\circ} \mathrm{C}$ (ethanollDMF). Anal. calcd. for $\mathrm{C}_{20} \mathrm{H}_{15} \mathrm{BrN}_{2} \mathrm{O}_{2}$ (395.24): C, 60.78; H, 3.83; Br, 20.22; N, 7.09. Found: C, 60.48; H, 3.53; Br, 20.02; N, 7.00.

2-(1H-Benzimidazol-2-yl)-3-[2-(4-bromophenyl)-1H-indol-3-yl]acrylonitrile 22

Brown powder. Yield (2.64 g, 60\%); m.p. $250-253^{\circ} \mathrm{C}$ (ethanollDMF). Anal. calcd. for $\mathrm{C}_{24} \mathrm{H}_{15} \mathrm{BrN}_{4}$ (439.30): C, 65.62; H, 3.44; Br, 18.19; N, 12.75. Found: C, 65.42; H, 3.24; Br, 18.09; N, 12.65.

\subsubsection{3-[2-(4-Bromophenyl)-1H-indol-3-yl]-5-oxopyrazolidine-4-carbonitrile 21}

A mixture of compound $20 \mathrm{~b}$ ( $3.95 \mathrm{~g}, 0.01 \mathrm{~mole})$ and hydrazine hydrate $(0.75 \mathrm{ml}, 0.015 \mathrm{~mole})$ in ethanol (20 ml) was refluxed for $3 \mathrm{~h}$, then poured into water. The resulting solid was collected and recrystallized from ethanol to give 21 as yellowish white crystals. Yield (1.91 g, 50\%); m.p.: 198-200 ${ }^{\circ} \mathrm{C}$. Anal. calcd for $\mathrm{C}_{18} \mathrm{H}_{13} \mathrm{BrN}_{4} \mathrm{O}$ 
(381.23): C, 56.71; H, 3.44; Br, 20.96; N, 14.70. Found: C, 56.51; H, 3.24; Br, 20.76; N, 14.50.

\subsubsection{4. $\{5$-[2-(4-Bromophenyl)-1H-indol-3-ylmethylene]-4-oxo-2-thioxo-1,3-thiazolidin-3-yl $\}$ acetic acid 23}

To a solution of rhodanine-3-acetic acid $(1.91 \mathrm{~g}, 0.01 \mathrm{~mol})$ and anhydrous sodium acetate $(0.5 \mathrm{~g})$ in glacial acetic acid was added the $1 \mathrm{H}$-indole-3-carboxaldehyde 4 ( $3 \mathrm{~g}, 0.01 \mathrm{~mol})$. The mixture was stirred under reflux for 6 $\mathrm{h}$ and then poured into ice-cold water. The precipitate was filtered, washed with water, dried and recrystallized from xylene to gives 23 as orange powder. Yield (3.08 g, 65\%); m.p.: 223-225 ${ }^{\circ} \mathrm{C}$. Anal. calcd. For $\mathrm{C}_{20} \mathrm{H}_{13} \mathrm{BrN}_{2} \mathrm{O}_{3} \mathrm{~S}_{2}$ (473.36): C, 50.75; H, 2.77; Br, 16.88; N, 5.92; S, 13.55. Found: C, 50.45; H, 2.57; Br, 16.58; N, 5.62; S, 13.25 .

\subsubsection{Ethyl 3-0xo-3-[(4-phenyl-1,3-thiazol-2-yl)amino]propanoate 25}

A mixture of an equimolar amount of 4-phenyl-2-aminothiazole 24 (1.76 g, $0.01 \mathrm{~mol})$ and diethylmalonate $1.6 \mathrm{~g}$, $0.01 \mathrm{~mol}$ ) was heated in an oil bath at $180^{\circ} \mathrm{C}$ for 2 hours then left to cool. The product was collected and used in second step.

\subsubsection{6-[(4-Phenyl-1,3-thiazol-2-yl)amino]-2-thioxo-2,3-dihydro-pyrimidin-4(5H)-one 26}

A mixture of ester $25(2.9 \mathrm{~g}, 0.01 \mathrm{~mol})$ and thiourea $(0.76 \mathrm{~g}, 0.01 \mathrm{~mol})$ in ethanol $(30 \mathrm{ml})$ containing sodium ethoxide was heated under reflux for $6 \mathrm{~h}$. The reaction mixture was poured into cold water and the formed solid product was collected by filtration, washed, dried and recrystallized from ethanol to gives $\mathbf{2 6}$ as yellow crystals. Yield (3.8 g, 60\%); m.p.:200-202 ${ }^{\circ} \mathrm{C}$. Anal. calcd. for $\mathrm{C}_{13} \mathrm{H}_{10} \mathrm{~N}_{4} \mathrm{O}_{2}$ (302.37): C, 51.64; H, 3.33; N, 18.53; $\mathrm{S}$, 21.21. Found: C, 51.44; H, 3.13; N, 18.33; S, 21.01.

\subsubsection{5-[2-(4-Bromophenyl)-1H-indol-3-ylmethylene]-6-[(4-phenyl-1,3-thiazol-2-yl)amino]- 2-thioxo-2,5-dihydropyrimidin-4(3H)-one 27}

A mixture of pyrimidine derivative 26 ( $3 \mathrm{~g}, 0.01 \mathrm{~mol})$ and $1 H$ - indole-3-carboxaldehyde 4 (3 g, $0.01 \mathrm{~mol})$ in ethanol $(30 \mathrm{ml})$ was heated under reflux for $6 \mathrm{~h}$., then left to cool. The solid product was collected by filtration and recrystallized from xylene to give 27 as green powder. Yield $\left(2.34 \mathrm{~g}, 40 \%\right.$. m.p.: 208-210 ${ }^{\circ} \mathrm{C}$. Anal. calcd. for $\mathrm{C}_{28} \mathrm{H}_{18} \mathrm{BrN}_{5} \mathrm{OS}_{2}$ (584.50): C, 57.54; H, 3.10; Br, 13.67; N,11.98; S,10.97. Found: C, 57.34; H, 3.00; Br, 13.47; N, 11.68; S, 10.67.

\subsubsection{4-[2-(4-Bromophenyl)-1H-indol-3-ylmethylene]-3-methyl-1-phenyl-1H-pyrazol-5(4H)-one 28}

A mixture of $1 H$-pyrazol-5(4H)-one $(1.74 \mathrm{~g}, 0.01 \mathrm{~mol})$ and $1 H$ - indole-3-carboxaldehyde 4 (3 g, $0.01 \mathrm{~mol})$ in acetic acid in the presence of anhydrous sodium acetate was refluxed for $5 \mathrm{~h}$. The reaction mixture was cooled to room temperature and poured into ice cold water. The solid separated out was filtered washed with water and recrystallized from (ethanol/DMF) to give 28 as orange crystals. Yield (3.01g, 66\%); m.p.: 190-192 ${ }^{\circ} \mathrm{C}$. Anal. calcd. for $\mathrm{C}_{25} \mathrm{H}_{18} \mathrm{BrN}_{3} \mathrm{O}$ (456.33): C, 65.80; H, 3.98; Br,17.51; N, 9.21. Found: C, 65.56; H, 3.68; Br, 17.31; N, 9.00 .

\subsubsection{2-(4-Bromophenyl)-3-(4-methyl-6-phenyl-2,6-dihydropyrazolo[3,4-c]pyrazol-3-yl)-}

$1 H$-indole 29a

A mixture of compound $28(4.56 \mathrm{~g}, 0.01 \mathrm{ml})$ and hydrazine hydrate $(0.5 \mathrm{ml}, 0.01 \mathrm{ml})$ in ethanol in present of few drops of acetic acid was refluxed for $7 \mathrm{~h}$. Reaction mixture was cooled at room temperature and poured in ice cold water. The solid separated was filtered, washed with water and recrystallized from ethanol to give 29a as yellow crystals. Yield (2.81 g, 60\%); m.p.: $180-182^{\circ} \mathrm{C}$. Anal.calcd. for $\mathrm{C}_{25} \mathrm{H}_{18} \mathrm{BrN}_{5}$ (468.34): C, 64.11; $\mathrm{H}$, 3.87; Br, 17.06; N, 14.95. Found: C, 64.00; H, 3.57; Br, 16.89; N, 14.65.

\subsubsection{2-(4-Bromophenyl)-3-(4-methyl-2,6-diphenyl-2,6-dihydropyrazolo[3,4-c]pyrazol-3-yl)- 1 -indole $29 \mathrm{~b}$}

A mixture of compound 28 (4.56 g, $0.01 \mathrm{ml})$, phenyl hydrazine $(1.08 \mathrm{ml}, 0.01 \mathrm{~mol})$, anhydrous sodium acetate $(0.5 \mathrm{~g})$ and acetic acid $(20 \mathrm{ml})$ was refluxed for $7 \mathrm{~h}$. Reaction mixture was cooled to room temperature and poured in ice cold water. The solid separated out was filtered, washed with water and recrystallized from ethanol 
to give 29b as yellow crystals. Yield (3.54 g, 65\%); m.p.: 105-106 ${ }^{\circ} \mathrm{C}$. Anal. calcd. for $\mathrm{C}_{31} \mathrm{H}_{22} \mathrm{BrN}_{5}$ (544.44): C, 68.39; H, 4.07; Br, 14.68; N, 12.86. Found: C, 68.09; H, 4.00; Br, 14.48; N, 12.66.

\subsubsection{3-(2-(4-Bromophenyl)-1H-indol-3-yl)-4-methyl- $N$-(4-methylphenyl)-6-phenyl-pyrazolo [3,4-c]-pyrazole-2 $(6 H)$-carbothioamide 30}

A mixture of compound 28 (4.56 g, $0.01 \mathrm{~mol})$ and $N$-(4-methylphenyl)thiosemicarbazide (1.81 g, $0.01 \mathrm{~mol})$ was refluxed in ethanol in the presence of $\mathrm{NaOH} / \mathrm{H}_{2} \mathrm{O}(10 \%, 5 \mathrm{ml})$ for $8 \mathrm{~h}$. Reaction mixture was cooled to room temperature and poured in ice-cold water. The solid separated out was filtered, washed with water and recrystallized from ethanol to give 30 as yellow crystals, Yield (3.89 g, 63\%); m.p.: $240-242^{\circ} \mathrm{C}$ (ethanol). Anal. Calcd. for $\mathrm{C}_{33} \mathrm{H}_{25} \mathrm{BrN}_{6} \mathrm{~S}$ (617.56): C, 64.18; H,4.08; Br,12.94; N, 13.61; S, 5.19. Found: C, 64.00; H, 4.00; Br, 12.70; N, 13.36; S, 5.09.

\subsubsection{4-(2-(4-Bromophenyl)-1H-indol-3-yl)-3-methyl-5-(4-nitrophenyl)-1-phenyl-1,5-dihydro- $6 H$-pyrazolo[3,4- $d]$ pyrimidine-6-thione 31}

A mixture of compound 28 (4.56 g, $0.01 \mathrm{~mol}), N$-(4-nitrophenyl)thiourea (1.97 g, $0.01 \mathrm{~mol})$ and potassium hydroxide $(0.5 \mathrm{~g})$ in ethanol $(20 \mathrm{ml})$ was refluxed with stirring for $4 \mathrm{~h}$. The reaction mixture was left overnight and then concentrated under reduced pressure. The solid residue was collected, washed with water and recrystallized from ethanol to gives 31 as orange powder, Yield (3.48 g, 55\%); m.p.: $150-152^{\circ} \mathrm{C}$. Anal. calcd. for $\mathrm{C}_{32} \mathrm{H}_{21} \mathrm{BrN}_{6} \mathrm{O}_{2} \mathrm{~S}$ (633.52): C, 60.67; H, 3.34; Br, 12.61; N, 13.27; S, 5.06. Found: 60.47; H, 3.14; Br, 12.41; N, 13.07; S, 4.89.

\subsection{Antimicrobial Assays}

Synthesized compounds 5c, 7, 9, 11, 13a, 27, 30 and 31 were screened for their antimicrobial activitiesin vitro against two species of Gram-positive bacteria, namely Staphylococcus saureus RCMB 0100010 (SA), Bacillus subtilis RCMB 010067 (BS) and two negative bacteria, namely Pseudomonas aeuroginosa RCMB 010043 (PA), Escherichia coli CMB 010052 (EC). Two fungal strains Aspergillus fumigatus RCMB 02568 (AF) and Candida albicans RCMB 05036 (CA) are used for antifungal activity. The antibacterial and antifungal activities were determined by means of inhibition $\% \pm$ standard deviation at a concentration of $100 \mu \mathrm{g} / \mathrm{ml}$ of tested samples [15] [16]. Optical densities of antimicrobial were measured after 24 hours at $37^{\circ} \mathrm{C}$ to bacteria and measured after 48 hours at $28^{\circ} \mathrm{C}$ to fungal using a multidetection microplate reader at the Regional Center for Mycology and Biotechnology (Sun Rise-Tecan, USA at $600 \mathrm{~nm}$ ) Al-Azhar University. Ampicillin, gentamicin were used as bacterial standards and amphotericin B was used as fungal standards for references to evaluate the efficacy of the tested compounds under the same conditions. The MICs of the compounds assays were determined by using microbroth kinetic system [17].

\section{Results and Discussion}

\subsection{Chemistry}

The synthesis of the new compounds is outlined in Schemes 1-6. 2-Substituted-indole reported to be obtained via Fischer indole synthesis using phenyl hydrazine and acetophenone derivatives 1a-d in present of polyphosphoric acid as catalysis [18]. Synthesis of $1 \mathrm{H}$-indole-3-carboxaldehyde derivative 4 from the 2-(4-bromophenyl)- $1 \mathrm{H}$-indole $3 \mathrm{c}$ via Vilsmeir Haack's formylation using phosphorus oxychloride $\left(\mathrm{POCl}_{3}\right)$ and $\mathrm{N}, \mathrm{N}^{\prime}$-dimethylformamide (DMF) [19] (Scheme 1). The IR spectrum of $\mathbf{4}$ revealed $\mathrm{C}=\mathrm{O}$ stretching band of formyl group at $1672 \mathrm{~cm}^{-1}$. $1 \mathrm{H}$ NMR spectrum showed an $\mathrm{D}_{2} \mathrm{O}$-exchangeable signal at $12.40 \mathrm{ppm}$ assigned to the $\mathrm{NH}$ proton and a non exchangeable signal at $\delta 9.95 \mathrm{ppm}$ corresponding to the formyl proton. The mass spectrum showed the molecular ion peak at $\mathrm{m} / \mathrm{z} 300$ corresponding to the molecular formula $\mathrm{C}_{15} \mathrm{H}_{10} \mathrm{BrNO}$.

The hydrazine derivatives 5a-c were obtained by the reaction of $1 H$-indole-3-carboxaldehyde derivative 4 with different substituted hydrazines [20] namely, hydrazine hydrate, phenyl hydrazine and 2-hydrazinyl-1,3benzothiazole (Scheme 1). The molecular structure of the synthesis compounds were established based on analytical and spectral data. For example, $1 \mathrm{H}$ NMR spectrum of compound $\mathbf{5 c}$ showed an $\mathrm{D}_{2} \mathrm{O}$-exchangeable signal at 7.95 and 11.82 assigned to the two $\mathrm{NH}$ protons and a non exchangeable signal at $8.42 \mathrm{ppm}$ corresponding $=\mathrm{CH}$ proton. On other hand mass spectrum of $5 \mathbf{c}$ showed a molecular ion peak $\mathrm{m} / \mathrm{z}$ at 447 corresponding to the molecular formula $\mathrm{C}_{22} \mathrm{H}_{15} \mathrm{BrN}_{4} \mathrm{~S}$. 
Reaction of $1 H$-indole-3-carboxaldehyde derivative $\mathbf{4}$ with different substituted acid hydrazides [21] such as 2-(quinolin-8-yloxy)acetohydrazide 6a, 2-(1H-indol-3-yl)acetohydrazide 6b, 4-(1H-indol-3-yl)butanehydrazide 6c and 1-benzofuran-2-carbohydrazide $\mathbf{6 d}$ in presence of catalytic amount of acetic acid in absolute ethanol<smiles>[R]C(=O)CCc1ccc(NN=C(C)C([R])=NCCc2ccc3cc([R])[nH]c3c2)cc1</smiles>

1

3

\begin{tabular}{|c|c|}
\hline $1,2,3$ & $\mathrm{R}$ \\
\hline $\mathrm{a}$ & $4-\mathrm{CH}_{3} \mathrm{C}_{6} \mathrm{H}_{4}$ \\
\hline $\mathrm{b}$ & $4-\mathrm{NH}_{2} \mathrm{C}_{6} \mathrm{H}_{4}$ \\
\hline $\mathrm{c}$ & $4-\mathrm{BrC}_{6} \mathrm{H}_{4}$ \\
\hline $\mathrm{d}$
\end{tabular}

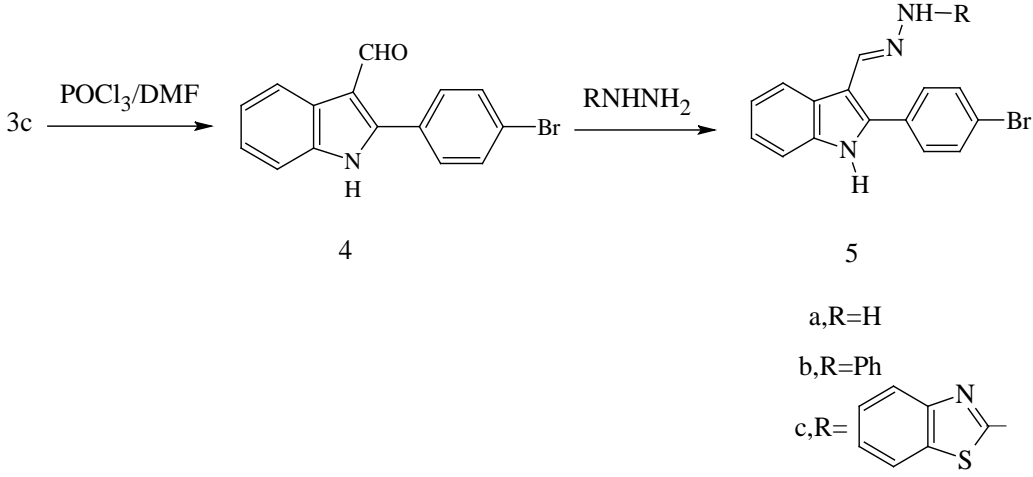

Scheme 1. Synthesis of compounds 3-5.

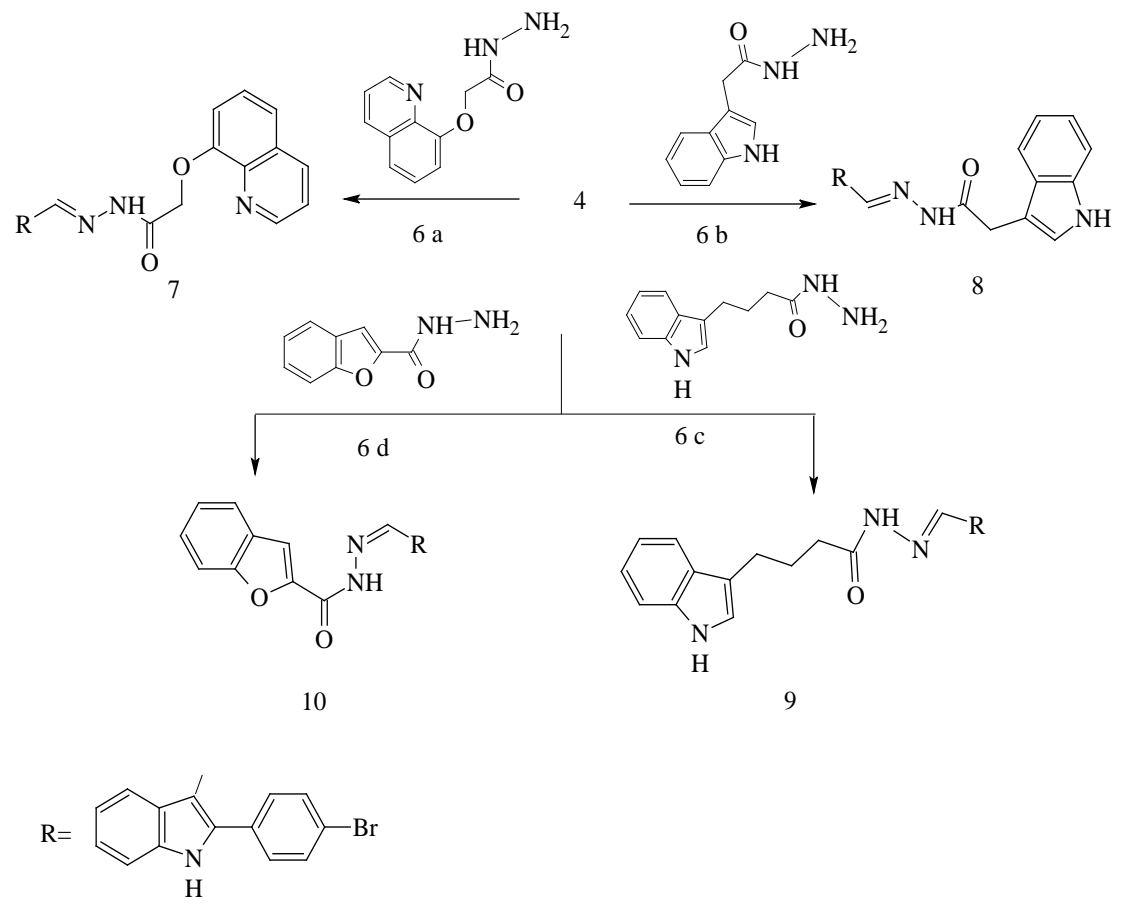

Scheme 2. Synthesis of compounds 7-10. 


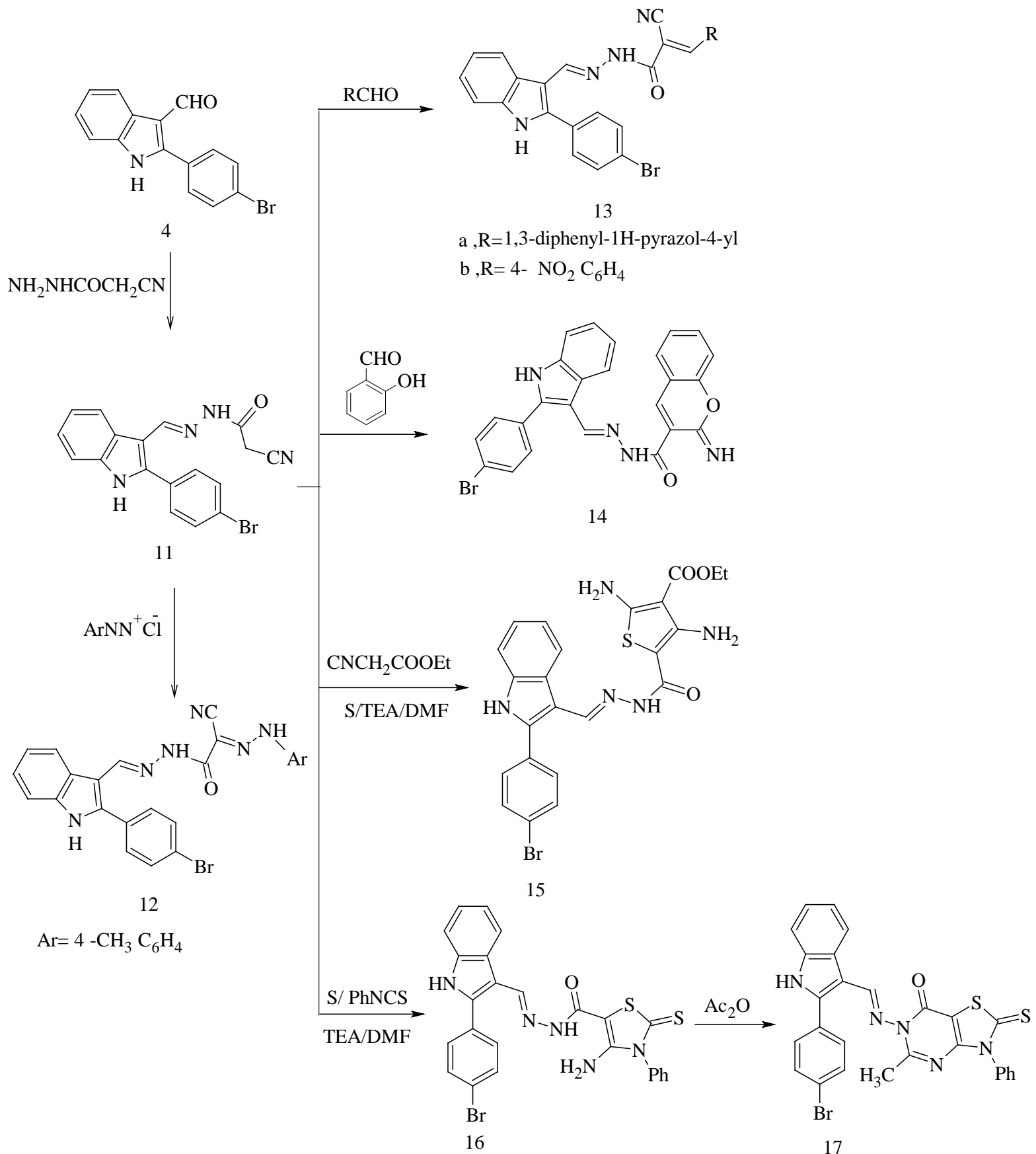

Scheme 3. Synthesis of compounds 11-17.

afforded the corresponding the acid hydrazide derivatives 7-10 respectively (Scheme 2). The assignment of the structure of the synthesis compounds were based on analytical and spectroscopic data. For example IR spectrum of 8 exhibit absorption band at $1604 \mathrm{~cm}^{-1}$ and $1654 \mathrm{~cm}^{-1}$ due to $-\mathrm{C}=\mathrm{N}$ and CO groups. 1H NMR of 8 exhibits signal at $\delta 8.90 \mathrm{ppm}$ for $=\mathrm{CH}$ proton and $\mathrm{D}_{2} \mathrm{O}$-exchangeable signal at $\delta 4.31$ and $12.03 \mathrm{ppm}$ assigned to the $2 \mathrm{NH}$ protons. The mass spectrum of compound 8 showed the molecular ion peak at $\mathrm{m} / \mathrm{z} 471$ corresponding to the molecular formula $\mathrm{C}_{25} \mathrm{H}_{19} \mathrm{BrN}_{4} \mathrm{O}$.

Reaction $1 \mathrm{H}$-indole-3-carboxaldehyde 4 with cyanoacetohydrazide in absolute ethanol [22] to form the $\mathrm{N}^{\prime}$ [1H-indol-3-ylmethylene]-2-cyanoacetohydrazide derivative 11. The assignment of the structure of compound 


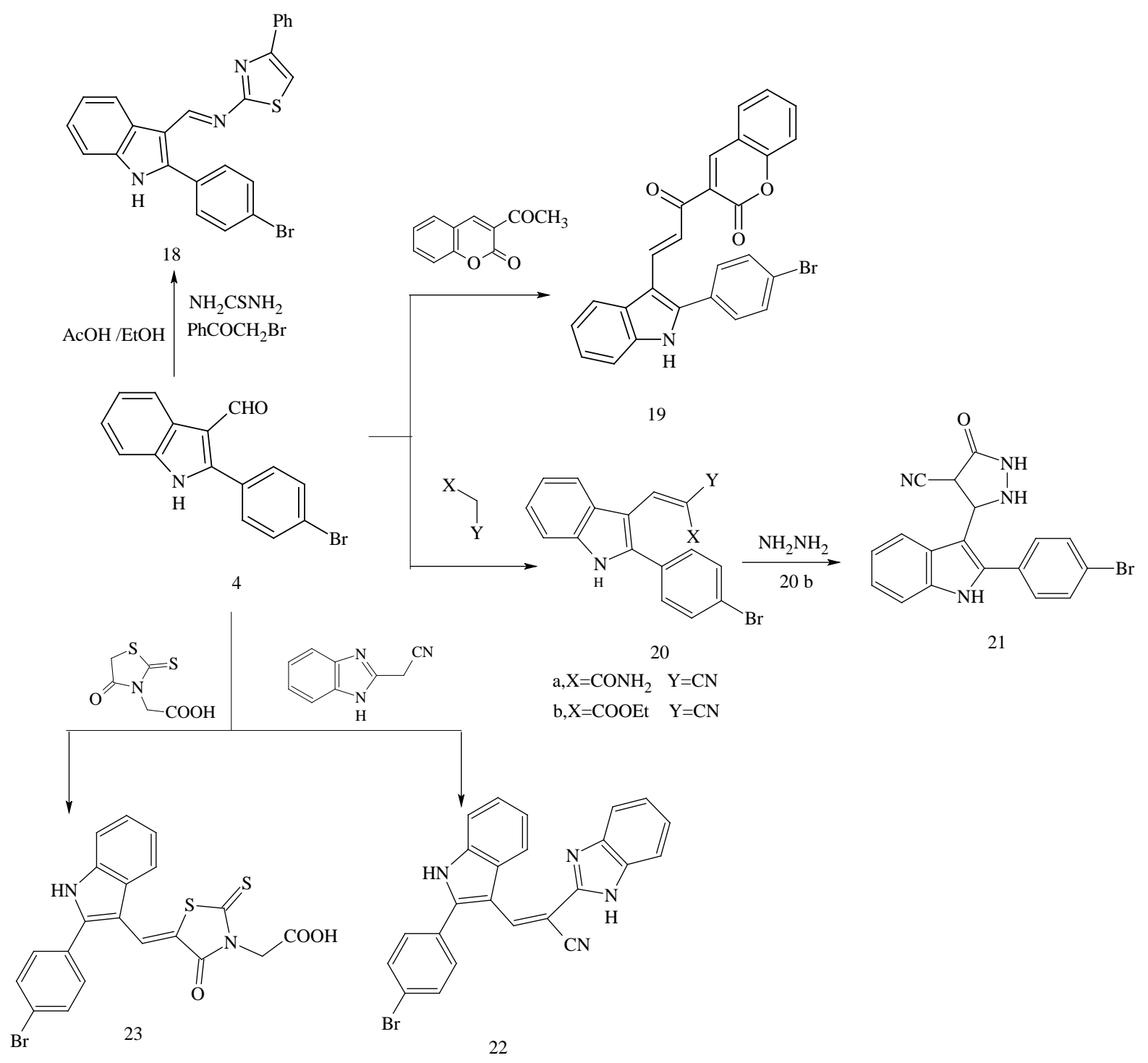

Scheme 4. Synthesis of compounds 18-23.

11 was based on analytical and spectroscopic data. Thus, the $1 \mathrm{H}$ NMR showed a singlet at $\delta 4.33$ for the $\mathrm{CH}_{2}$ group, a singlet at $\delta$ 8.33ppm for the $=\mathrm{CH}$ proton and $\mathrm{D}_{2} \mathrm{O}$-exchangeable single at $\delta 11.33,11.93 \mathrm{ppm}$ for the two NH protons. ${ }^{13} \mathrm{C}$ NMR spectrum of 11 displayed signals at $\delta 24.39,116.19,157.73$ and $163.58 \mathrm{ppm}$ for $\mathrm{CH}_{2}$, $\mathrm{CN}, \mathrm{C}=\mathrm{N}$ and $\mathrm{CO}$ respectively. Further structure elucidation of compound $\mathbf{1 1}$ was obtained through the study of its reactivity towards chemical reagents. Thus, the reaction of $\mathbf{1 1}$ with 4-methylbenzene diazonium chloride [23] gave the hydrazone derivatives 12 (Scheme 3). The structures of the compound 12 were determined from spectroscopic and elemental analytical data (see Experimental section).

Knoevengel condensation of the 2-cyanoacetohydrazide derivatives $\mathbf{1 1}$ with aromatic aldehydes namely 1,3-diphenyl-1H-pyrazole-4-carboxaldehyde and $p$-nitrobenzaldehyde [24] afforded benzylidene derivatives 13a, b (Scheme 3). The IR spectrum of compound 13a, taken as a typical example of the series prepared, revealed absorption bands at $1686 \mathrm{~cm}^{-1}, 2204 \mathrm{~cm}^{-1}, 3335 \mathrm{~cm}^{-1}$ and $3126 \mathrm{~cm}^{-1}$ corresponding to carbonyl, nitrile and 2 $\mathrm{NH}$ groups, respectively. Where the $1 \mathrm{H}$ NMR spectra showed the absence of the active methylene proton and showed signals at $\delta 9.21 \mathrm{ppm}$ for $\mathrm{N}=\mathrm{CH}$ proton and $\mathrm{D}_{2} \mathrm{O}$-exchangeable signal at $\delta 11.73$ and 12.04 assigned to the $2 \mathrm{NH}$ protons. Its mass spectrum showed a molecular ion peak at $\mathrm{m} / \mathrm{z} 611$ corresponding to the molecular formula $\mathrm{C}_{34} \mathrm{H}_{23} \mathrm{BrN}_{6} \mathrm{O}$.

Cyclocondensation of 2-cyanoacetohydrazide derivatives $\mathbf{1 1}$ with salicyaldehyde in dioxane in the presence of 


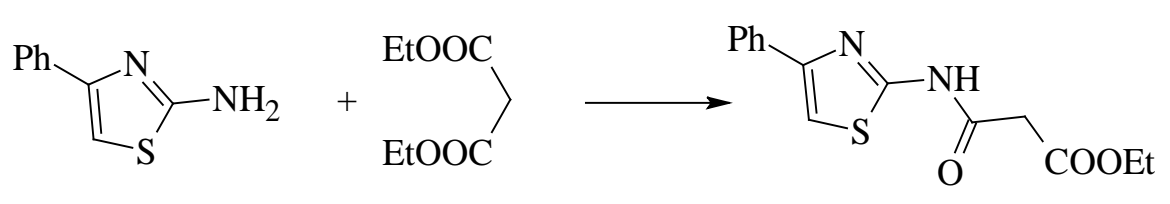

24

25

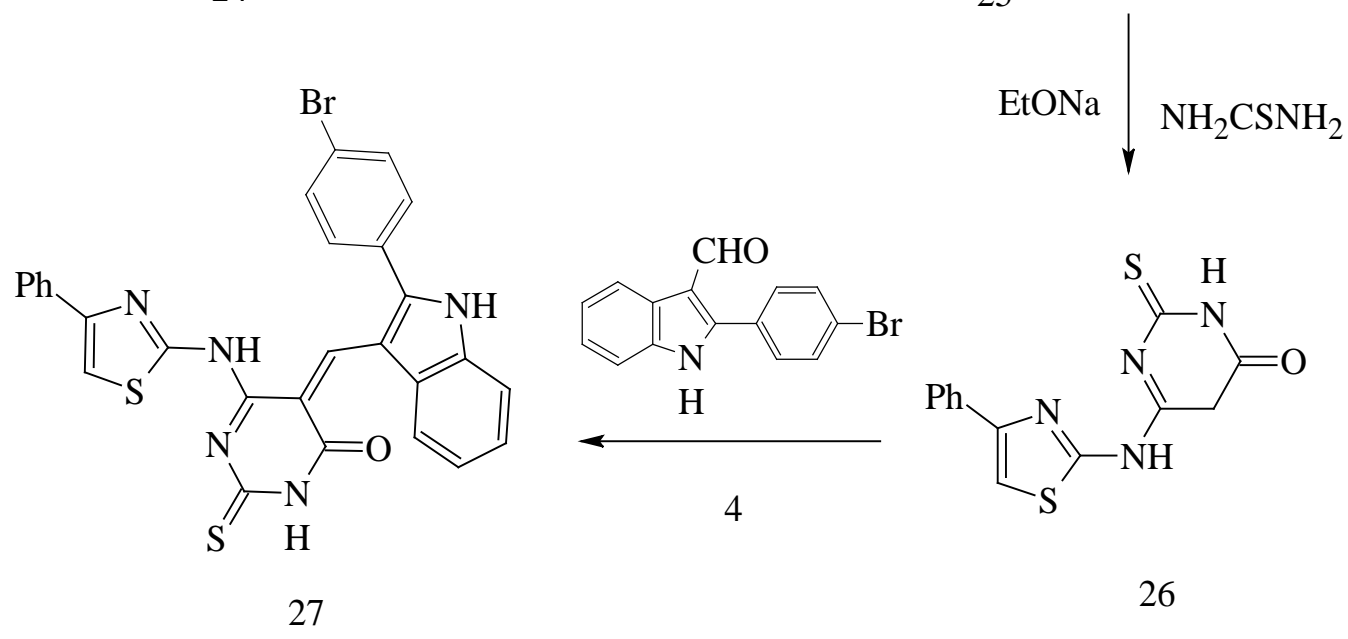

Scheme 5. Synthesis of compounds 25-27.

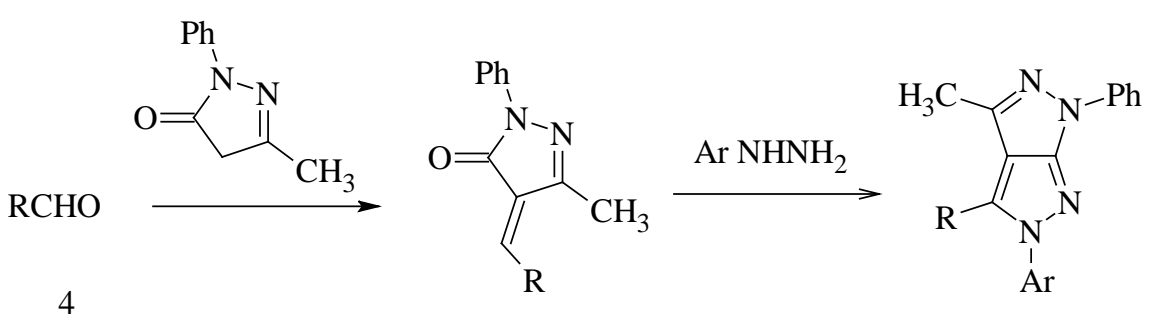

28

29

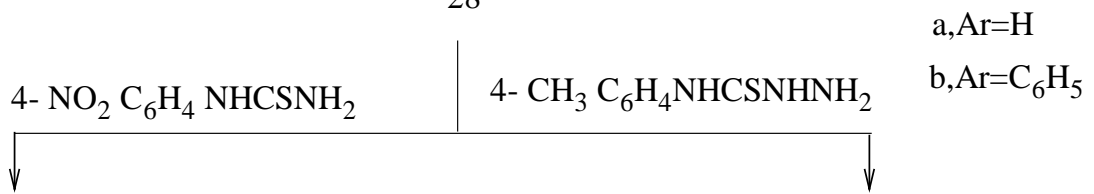<smiles>[R]c1c2c(C)nn(-c3ccccc3)c2nc(=S)n1-c1ccc([N+](=O)[O-])cc1</smiles>

31<smiles></smiles><smiles>[R]c1c2c(C)nn(-c3ccccc3)c2nn1C(=S)Nc1ccc(C)cc1</smiles>

30

Scheme 6. Synthesis of compounds 28-31. 
a catalytic amount of piperidine afforded 2-imino-2H-chromene-3-carbohydrazide $\mathbf{1 4}$. The plausible mechanism for the formation of compound $\mathbf{1 4}$ may be attributed to the initial Knoevenagel condensation of the active methylene nitrile of $\mathbf{1 1}$ with carbonyl group of salicyaldehyde followed by an intramolecular 1,6-dipolar cyclization via the addition of the phenolic OH group to the cyano function to afford the target compounds [25] (Figure 1). $1 \mathrm{H}$ NMR spectrum of 14 showed three $\mathrm{D}_{2} \mathrm{O}$-exchangeable signal at $\delta 11.44, \delta 11.56$ and $\delta 12.14 \mathrm{ppm}$ due to three NH protons. Its mass spectrum showed a molecular ion peak at $\mathrm{m} / \mathrm{z} 485$ corresponding to the molecular formula $\mathrm{C}_{25} \mathrm{H}_{17} \mathrm{BrN}_{4} \mathrm{O}_{2}$.

The reaction of $\mathbf{1 1}$ with ethyl cyanoacetate and elemental sulfur in the presence of triethylamine gave the thiophene derivatives [26] 15. The structure of compound $\mathbf{1 5}$ was confirmed by its infrared spectrum which indicated the absence of $\mathrm{CN}$ absorption band and contain the characteristic absorption bands for NH and CO functional groups. On the other hand the reaction of $\mathbf{1 1}$ with elemental sulfur and phenylisothiocyanate [27] gave the thiazole derivative 16. Compounds 15 and $\mathbf{1 6}$ were obtained according to the proposed following mechanism (Figure 2). The structure of compounds 15 and $\mathbf{1 6}$ were elucidated on the basis of elemental analysis and spectral data. The IR spectrum of thiazoline $\mathbf{1 6}$ revealed the absence of $\mathrm{CN}$ absorption band and the presence of new absorption bands at 3395, $3269 \mathrm{~cm}^{-1}$ assignable to $\mathrm{NH}_{2}$ group and band at $1237 \mathrm{~cm}^{-1}$ due to $\mathrm{C}=\mathrm{S}$ group. The ${ }^{13} \mathrm{C}$ NMR data showed signals at $\delta 183.91, \delta 161.36, \delta 153$ and $\delta 147.39 \mathrm{ppm}$ to CS, CO, C-NH $\mathrm{N}_{2}$ and C=N. Cyclization of thiazoline 16 with acetic anhydride afforded 1,3-thiazolo[4,5-d]pyrimidin-7(6H)-one derivative $\mathbf{1 7}$ (Scheme $3)$.

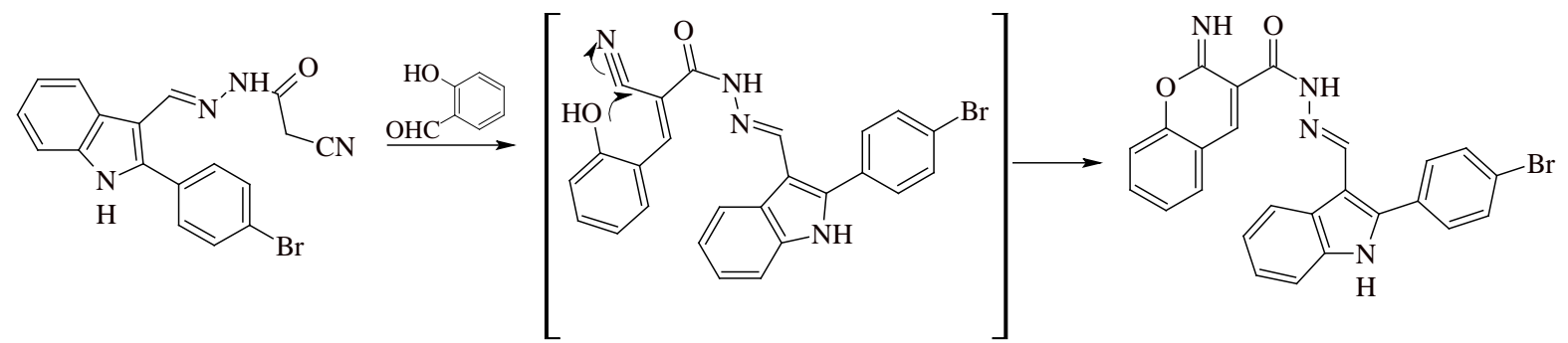

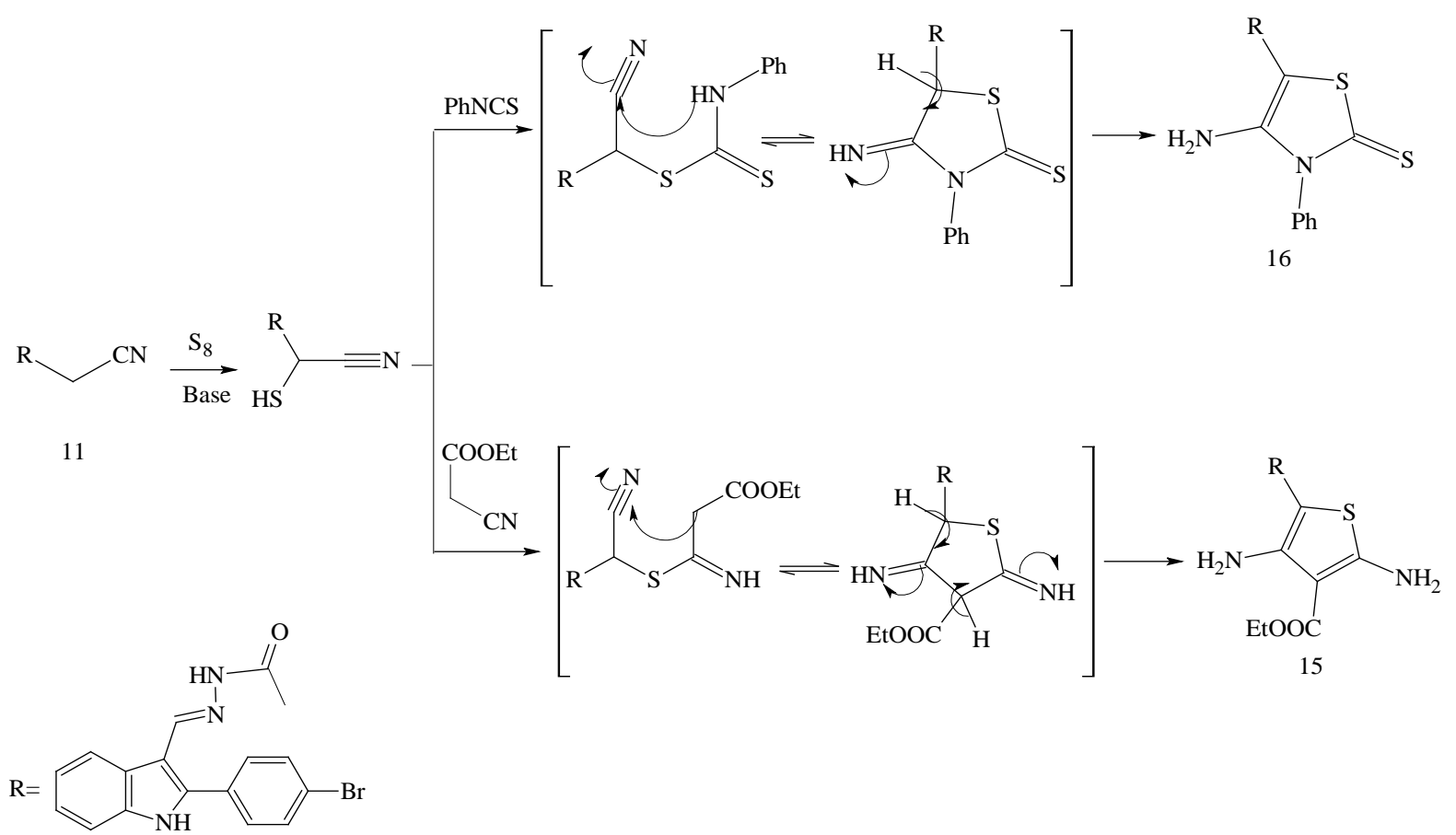

Figure 2. Proposed mechanism of formation of compounds $\mathbf{1 5}$ and $\mathbf{1 6 .}$ 
$N$-[1H-indol-3-ylmethylene]-1,3-thiazol-2-amine 18 was synthesized by the one-pot three compounds. Thus, condensation of phenacyl bromide, $1 \mathrm{H}$-indole-3-carboxaldehyde $\mathbf{4}$ and thiourea [28] under conventional heating in absolute ethanol using catalytic amount of acetic acid. The 1H NMR spectra of compound $\mathbf{1 8}$ showed the absence of the aldehyde proton, moreover $\mathrm{D}_{2} \mathrm{O}$-exchangeable signal at $\delta 12.43 \mathrm{ppm}$ due to the $\mathrm{NH}$ proton and signal at $\delta 6.85 \mathrm{ppm}$ for $=\mathrm{CH}$ proton. Condensation of $1 \mathrm{H}$-indole-3-carboxaldehyde 4 with 3-acetyl- $2 \mathrm{H}$-chromen2-one [29] afforded 3-[3-(1H-indol-3-yl)prop-2-enoyl]-2H-chromen-2-one 19 (Scheme 4).

Condensation of $\mathbf{4}$ with cyanoacetamide, ethyl cyanoacetate and $1 H$-benzimidazol-2-yl-acetonitrile [30] afforded 3-(1H-indol-3-yl)-2-cyanoprop-2-enamide, ethyl 3-(1H-indol-3-yl)-2-cyanoprop-2-enoate 20a,b and 2(1H-benzimidazol-2-yl)-3-(1H-indol-3-yl)crylonitrile 22 respectively (Scheme 4). The structure of the reaction product 20a,b and 22 were ascertained on the basis of its elemental analysis and spectral data. The IR spectrum of compound 20a exhibited characteristic absorption bands at $3466 \mathrm{~cm}^{-1}, 3310 \mathrm{~cm}^{-1}, 2203 \mathrm{~cm}^{-1}$ and $1688 \mathrm{~cm}^{-1}$ corresponding to $\mathrm{NH}_{2}, \mathrm{CN}$ and $\mathrm{CO}$ groups respectively. The $1 \mathrm{H}$ NMR spectrum of 20a indicated the presence of one singlet peak at $\delta 8.22 \mathrm{ppm}$ of the $=\mathrm{CH}$ proton and the disappearance of a singlet at $\delta 9.95 \mathrm{ppm}$ of CHO proton. Cyclization of 20b by hydrazine hydrate to 5-oxopyrazolidine-4-carbonitrile derivative $\mathbf{2 1}$ was achieved by refluxing in ethanol. The $1 \mathrm{H}$ NMR spectrum of compound 21 indicated the presence of $\mathrm{D}_{2} \mathrm{O}$-ex- changeable singlet at $\delta 10.18, \delta 11.41$ and $\delta 12.22 \mathrm{ppm}$ which correspond to three $\mathrm{NH}$ groups.

On the other hand, the reaction of $1 H$-indole-3-carboxaldehyde 4 with rhodanine-3-acetic acid [31] afforded [5-(1H-indol-3-ylmethylene)-1,3-thiazolidin-3-yl]acetic acid derivative 23. The 1H-NMR spectrum of compound 23 indicated the presence of singlet at $\delta 4.71 \mathrm{ppm}$ of the $\mathrm{CH}_{2}$ and $\mathrm{D}_{2} \mathrm{O}$-exchangeable singlet at $\delta 12.60$ $\mathrm{ppm}$ of the $\mathrm{OH}$ proton. Mass spectrum of 23 showed a molecular ion peak $\mathrm{m} / \mathrm{z}$ at 473 corresponding to the molecular formula $\mathrm{C}_{20} \mathrm{H}_{13} \mathrm{BrN}_{2} \mathrm{O}_{3} \mathrm{~S}_{2}$ (Scheme 4).

The key substrate ester $\mathbf{2 5}$ was synthesized from the reaction of 4-phenyl-2-amino-thiazole $\mathbf{2 4}$ and diethyl malonate. Reaction of ester $\mathbf{2 5}$ with thiourea in ethanolic sodium ethoxide solution afforded 6-[1,3-thiazol2-ylamino]-2-thioxo-2,3-dihydropyrimidin-4(5H)-one derivative [32] 26. Treatment of pyrimidinone derivative 26 with $1 H$-indole-3-carboxaldehyde 4 afforded 5-[1H-indol-3-ylmethylene]-2-thioxo-2,5-dihydropyrimidin4(3H)-one 27 (Scheme 5). The structure of $\mathbf{2 7}$ was identified as the reaction product on the basis of its elemental analysis and spectroscopic data. The $1 \mathrm{H}$ NMR spectrum of compound 27 indicated the presence of singlet signal at $\delta 9.96 \mathrm{ppm}$ of the $=\mathrm{CH}$ proton and $\mathrm{D}_{2} \mathrm{O}$-exchangeable singlet at $\delta 12.18, \delta 12.22$ and $\delta 12.40 \mathrm{ppm}$ corresponding to the three $\mathrm{NH}$ protons. ${ }^{13} \mathrm{C}$ NMR spectrum showed signal at $\delta 185.37(\mathrm{C}=\mathrm{S})$ and $168.01(\mathrm{C}=\mathrm{O})$.

Condensation of $1 \mathrm{H}$-indole-3-carboxaldehyde 4 with 5-methyl-2-phenyl-2,4-dihydro-3H-pyrazol-3-one afforded 4-[1H-indol-3-ylmethylene]-1H-pyrazol-5(4H)one 28 (Scheme 6). The IR spectrum of 28 exhibited characteristic absorption bands at $3275 \mathrm{~cm}^{-1}$ and $1706 \mathrm{~cm}^{-1}$ corresponding to $\mathrm{NH}$ and $\mathrm{CO}$ groups, respectively. ${ }^{13} \mathrm{C}$ NMR spectrum showed signal at $\delta 12.58\left(\mathrm{CH}_{3}\right), \delta 153(\mathrm{C}=\mathrm{C})$ and $\delta 160(\mathrm{C}=\mathrm{O})$. Mass spectrum of 28 showed a molecular ion peak $m / z$ at 456 corresponding to the molecular formula $\mathrm{C}_{25} \mathrm{H}_{18} \mathrm{BrN}_{3} \mathrm{O}$.

Compound 28 was used as key intermediates in the synthesis of novel pyrazolo[3,4-c]pyrazolone and pyrazole[3.4-d] pyrimidine derivatives via their interaction with different reagents. Thus, the reaction of $\mathbf{2 8}$ with hydrazine hydrate, phenyl hydrazine and $N$-(4-methylphenyl)thiosemicarbazide by cyclocondensation reaction [33] afforded 3-(4-methyl-pyrazolo[3,4-c]pyrazol-3-yl)-1H-indole derivatives 29a,b and pyrazolo[3,4-c]-pyrazole2(6H)-carbothioamide 30 respectively (Scheme 6 ). The structure of the newly synthesis compounds were based on their correct elemental analysis and spectral data. 1H NMR spectrum of 30 exhibited a singlet signal at $\delta 2.28$ ppm due to $\mathrm{CH}_{3}$ protons of tolyl moiety. Its mass spectrum, the compound displayed the molecular ion peak at $\mathrm{m} / \mathrm{z} 617$ corresponding to the molecular formula $\mathrm{C}_{33} \mathrm{H}_{25} \mathrm{BrN}_{6} \mathrm{~S}$. Alternatively, treatment of the compound 28 with $N$-(4-nitrophenyl)thiourea [34] afforded pyrazolo[3,4-d]pyrimidine-6-thione derivatives 31. The structures of the compound 31 were determined from spectroscopic and elemental analytical data (see Experimental section).

\subsection{Antimicrobial Activity}

The newly synthesized compounds 5c, 7, 9, 11, 13a, 27, 30 and 31 were evaluated for their in vitro antibacterial activity against Gram-positive namely Staphylococcus aureus RCMB 0100010 (SA) and Bacillus subtilis RCMB 010067 (BS) and Gram-negative Pseudomonas aeuroginosa RCMB 010043 (PA) and Escherichia coli RCMB 010052 (EC). They were also evaluated for their in vitro antifungal activity against Aspergillus fumigatus RCMB 02568 (AF) and Candida albicans RCMB 05036 (CA). Ampicillin was the standard used for the evaluation of antibacterial activity against gram positive bacteria and Gentamicin was used as a standard in assessing the activity of the tested compounds against gram negative bacteria, while Amphotericin B was taken as 
a reference for the antifungal effect. The inhibitory effects of the synthetic compounds against these organisms are given in Table 2, Figure 3 and Figure 4.

In general, most of the tested compounds revealed better activity against the Gram-positive rather than the Gram-negative bacteria. All test compounds were found to be inactive against Pseudomonas aeuroginosa RCMB 010043 (PA). It was shown (Figure 3) that the majority of the compounds studied possessed significant antibacterial activity towards Staphylococcus aureus RCMB 0100010 (SA), Bacillus subtilis RCMB 010067 (BS) and Escherichia coli RCMB 010052 (EC). The highest activities were observed for compounds 9 and $\mathbf{3 0 ,}$ followed by 11, 13a and 31.Compounds $5 c, 7$ and 27 showed the least antibacterial activity.

It was shown (Figure 4) that the compounds 5c, 11, 31 strong antifunger activity against Aspergillus fumigates RCMB 02568 (AF) and Candida albicans RCMB 05036 (CA) comparable to Amphotericin B. The com-

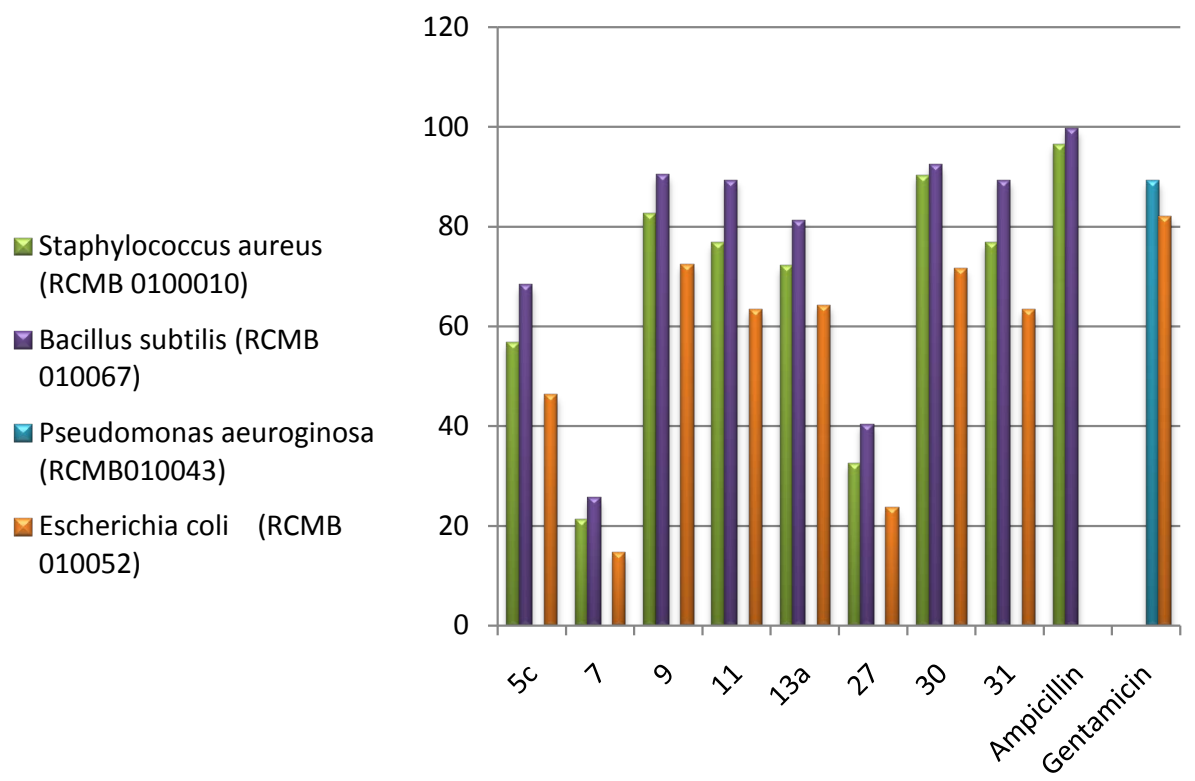

Figure 3. Graphical representation of the antibacterial activity of tested compounds compared to Ampicillin and Gentamicin.

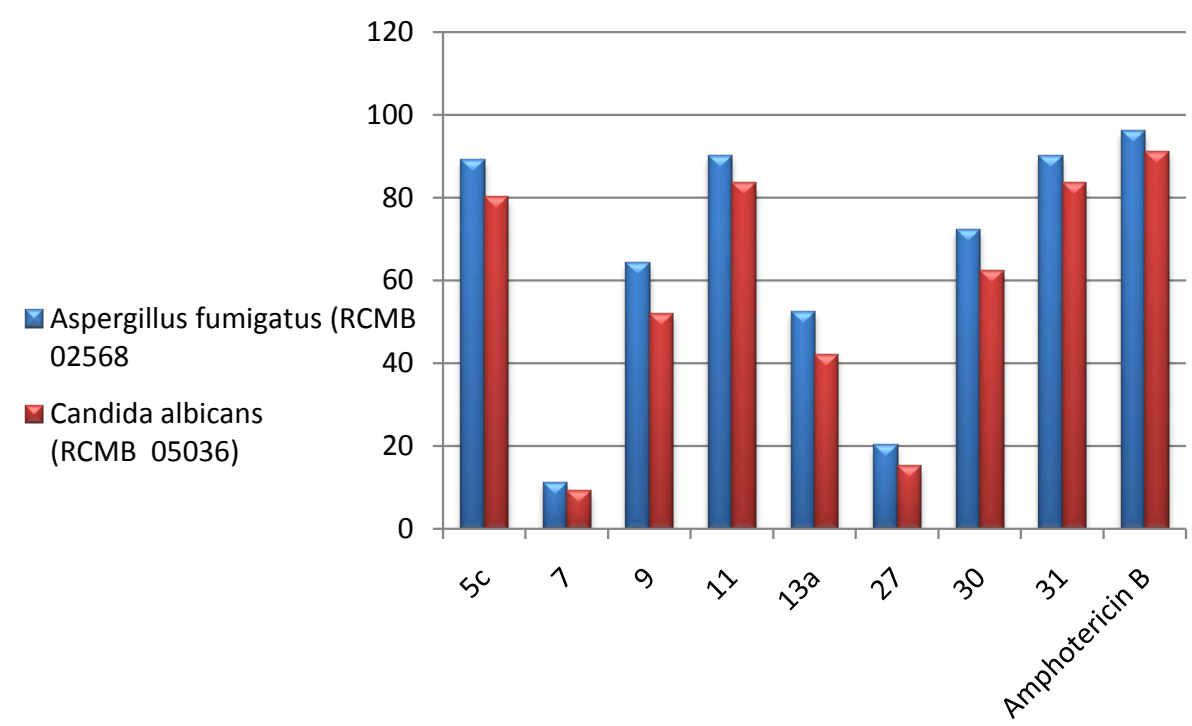

Figure 4. Graphical representation of the antifungal activity of tested compounds compared to Amphotericin B. 
pounds 9, 13a and 30 showed moderate activities against Aspergillus fumigates RCMB 02568 (AF) and Candida albicans RCMB 05036 (CA) comparable to Amphotericin B. While the compounds $\mathbf{7}$ and 27 weak antifungal activity against Aspergillus fumigates RCMB 02568 (AF) and Candida albicans RCMB 05036 (CA) comparable to Amphotericin B.

The minimum inhibitory concentration (MIC) was considered to be the lowest concentration of the tested compound which inhibits growth of the microorganisms. The initial screening of the tested compounds showed promising activity of the compounds $\mathbf{5 c}, \mathbf{9}, \mathbf{3 0}$ and $\mathbf{3 1}$ which encouraged the determination of their minimum inhibitory concentration (MIC) (Table 3).

The best results were demonstrated by compounds $\mathbf{9 ,} \mathbf{3 0}$ and $\mathbf{3 1}$ as antibacteria, it possessed double the activity of the standard, Ampicillin against Bacillus subtilis RCMB 010067 (BS) 1.95 and $3.9 \mu \mathrm{g} / \mathrm{ml}$ respectively. Moderate activity against Staphylococcus aureus RCMB 0100010 (SA) and Escherichia coli RCMB 010052 (EC) were also demonstrated by compounds 9 and $\mathbf{3 0}$. On other hand moderate activity against Aspergillus fumigatus RCMB 02568 (AF) and Candida albicans RCMB 05036 (CA) were also demonstrated by compounds 5c and 31.

Table 2. Antimicrobial evaluation of the some synthesized compounds.

\begin{tabular}{|c|c|c|c|c|c|c|}
\hline \multirow{3}{*}{ Comp. No. } & \multicolumn{4}{|c|}{ Inhibition $\% \pm$ standard deviation } & & \\
\hline & \multicolumn{2}{|c|}{ Gram positive bacteria } & \multicolumn{2}{|c|}{ Ggram negative e bacteria } & \multicolumn{2}{|l|}{ Fungal } \\
\hline & SA & BS & PA & ES & $\mathrm{AF}$ & CA \\
\hline $5 c$ & $56.85 \pm 0.58$ & $68.32 \pm 1.2$ & NA & $46.32 \pm 0.58$ & $89.25 \pm 0.72$ & $80.23 \pm 1.2$ \\
\hline 7 & $21.25 \pm 0.58$ & $25.63 \pm 0.28$ & NA & $14.63 \pm 0.2$ & $11.22 \pm 0.28$ & $9.32 \pm 0.72$ \\
\hline 9 & $82.63 \pm 0.75$ & $90.42 \pm 0.28$ & NA & $72.46 \pm 0.28$ & $64.35 \pm 0.58$ & $52.14 \pm 0.63$ \\
\hline 11 & $76.8 \pm 0.58$ & $89.4 \pm 0.63$ & NA & $63.5 \pm 0.72$ & $90.3 \pm 0.58$ & $83.6 \pm 0.28$ \\
\hline 13a & $72.14 \pm 0.58$ & $81.32 \pm 0.63$ & NA & $64.21 \pm 0.63$ & $52.63 \pm 0.58$ & $42.18 \pm 1.2$ \\
\hline 27 & $32.6 \pm 0.63$ & $40.4 \pm 0.85$ & NA & $23.6 \pm 1.2$ & $20.3 \pm 1.2$ & $15.4 \pm 0.58$ \\
\hline 30 & $90.3 \pm 1.2$ & $92.4 \pm 0.72$ & NA & $71.6 \pm 0.93$ & $72.4 \pm 0.58$ & $62.5 \pm 0.28$ \\
\hline 31 & $76.8 \pm 0.58$ & $89.4 \pm 0.63$ & NA & $63.5 \pm 0.72$ & $90.3 \pm 0.58$ & $83.6 \pm 0.28$ \\
\hline Ampicillin & $96.52 \pm 0.2$ & $99.65 \pm 0.3$ & & & & \\
\hline Gentamicin & & & $89.23 \pm 0.1$ & $82.14 \pm 0.3$ & & \\
\hline Amphotericin B & & & & & $96.25 \pm 0.1$ & $91.29 \pm 0.1$ \\
\hline
\end{tabular}

(SA): Staphylococcus aureus RCMB 0100010, (BS): Bacillus subtilis RCMB 010067 (BS), (PA): Pseudomonas aeuroginosa RCMB 010043, (EC): Escherichia coli RCMB 010052, (AF): Aspergillus fumigatus RCMB02568 and (CA): Candida albicans RCMB 05036.

Table 3. Minimum inhibitory concentration of compounds 5c, 9, 29 and 31.

\begin{tabular}{|c|c|c|c|c|c|c|}
\hline \multirow{3}{*}{$\begin{array}{c}\text { Comp. } \\
\text { No. }\end{array}$} & \multicolumn{4}{|c|}{ Minimum inhibitory concentration ( $\mu \mathrm{g} / \mathrm{ml})$} & & \\
\hline & \multicolumn{2}{|c|}{ Gram positive bacteria } & \multicolumn{2}{|c|}{ Gram negative e bacteria } & \multicolumn{2}{|l|}{ Fungi } \\
\hline & SA & BS & PA & EC & $\mathrm{AF}$ & CA \\
\hline $5 c$ & 31.25 & 15.63 & NA & 62.5 & 3.9 & 3.9 \\
\hline 9 & 3.9 & 1.95 & NA & 7.81 & 15.63 & 31.25 \\
\hline 30 & 1.95 & 1.95 & NA & 7.81 & 7.81 & 15.63 \\
\hline 31 & 7.81 & 1.95 & NA & 15.63 & 1.95 & 3.9 \\
\hline Ampicillin & 0.98 & 3.9 & & & & \\
\hline Gentamicin & & & 1.95 & 3.9 & & \\
\hline Amphotericin B & & & & & 0.98 & 1.95 \\
\hline
\end{tabular}




\section{Conclusion}

In the present work, we synthesized novel series of 3-substituted indole by reaction of indole-3-carboxaldehyde derivative with different reagents. Screening for some selected compounds was carried for their potential antibacterial, antifungal activity. Most of the tested compounds revealed better activity against the Gram-positive rather than the Gram negative bacteria. All test compounds were found to be inactive against Pseudomonas aeuroginosa. Compounds 9, 30 and 31 exhibited excellent activity against Staphylococcus aureus, Bacillus subtilis and Escherichia coli compared with the standards drugs, while compounds 5c, $\mathbf{1 1}$ and $\mathbf{3 1}$ have strong antifunger activity against Aspergillus fumigatus and Candida albicans comparable to Amphotericin B.

\section{References}

[1] Nagai, K., Davies, T.A., Jacobs, M.R. and Appelbaum, P.C. (2002) Effects of Amino Acid Alterations in PenicillinBinding Proteins (Pbps) 1a, 2b, and 2× on PBP Affinities of Penicillin, Ampicillin, Amoxicillin, Cefditoren, Cefuroxime, Cefprozil, and Cefaclor in 18 Clinical Isolates of Penicillin-Susceptible, Intermediate, and Resistant Pneumococci. Antimicrobial Agents and Chemotherapy, 46, 1273-1280. http://dx.doi.org/10.1128/AAC.46.5.1273-1280.2002

[2] Kaniwa, K., Arai, M.A., Li, X. and Ishibashi, M. (2007) Synthesis, Determination of Stereochemistry, and Evaluation of New Bisindole Alkaloids from the Myxomycete Arcyriaferruginea: An Approach for Wnt Signal Inhibitor. Bioorganic \& Medicinal Chemistry Letters, 17, 4254-4257. http://dx.doi.org/10.1016/j.bmcl.2007.05.033

[3] Mingxing Teng, M. (2014) Total Synthesis of the Monoterpenoid Indole Alkaloid ( \pm )-Aspidophylline A. Angewandte Chemie International Edition, 53, 1814-1817.

[4] Pilar, M.F., Pedro, M. and Juan, A.B. (2001) Synthesis of the Indole Alkaloids Meridianins from the Tunicate Aplidium Meridianum. Tetrahedron, 57, 2355-2363. http://dx.doi.org/10.1016/S0040-4020(01)00102-8

[5] Sharma, A. and Pathak, D.M. (2013) The Synthesis and Antimicrobial Activity of Indole Thiacarbamide Derivatives. International Journal of Scientific \& Engineering Research, 4, 203-206.

[6] Chavan Rajashree, S. and More Harinath, N. (2011) Synthesis, Characterization and Evaluation of Analgesic and AntiInflammatory Activities of Some Novel 2-(4,5-Dihydro-1H-pyrazol-3-yl)-3-phenyl- $1 H$-indole. Journal of Pharmacy Research, 4, 1575-1578.

[7] Kawasaki, I., Katsuma, H., Nakayama, Y., Yamashita, M.Y. and Ohta, S. (1998) Total Synthesis of Topsentin, Antiviral and Antitumor Bis(indolyl)imidazole. Heterocycles, 48, 1887-1901. http://dx.doi.org/10.3987/COM-98-8225

[8] Kawasaki, I., Yamashita, M.Y. and Ohta, S. (1996) Total Synthesis of Nortopsentins A-D, Marine Alkaloids. Chemical and Pharmaceutical Bulletin, 44, 1831-1839. http://dx.doi.org/10.1248/cpb.44.1831

[9] Sun, H.H. and Sakemi, J. (1991) A Brominated (Aminoimidazolinyl)indole from the Sponge Discodermia Polydiscus. The Journal of Organic Chemistry, 56, 4307-4308. http://dx.doi.org/10.1021/jo00013a045

[10] Raju, R., Gromyko, O., Fedorenko, V., Luzhetskyy, A. and Müler, R. (2012) Pimprinols A-C, from the Terrestrial Actinomycete, Streptomyces sp. Tetrahedron Letters, 53, 3009-3011. http://dx.doi.org/10.1016/j.tetlet.2012.03.134

[11] Fresneda, P.M., Castaneda, M., Blug, M. and Molina, P. (2007) Iminophosphorane-Based Preparation of 2,5-Disubstituted Oxazole Derivatives: Synthesis of the Marine Aalkaloid Almazole C. Synlett, 2, 324-326. http://dx.doi.org/10.1055/s-2007-967995

[12] Aaron, M.S., Richard, A.L. and David, C.R. (2007) Bacillamides from a Hypersaline Microbial Mat Bacterium. Journal of Natural Products, 70, 1793-1795. http://dx.doi.org/10.1021/np070126a

[13] Gao, X., Chooi, Y.-H., Ames, B.D., Wang, P., Walsh, C.T. and Tang,Y. (2011) Fungal Indole Alkaloid Biosynthesis: Genetic and Biochemical Investigation of the Tryptoquialanine Pathway in Penicillium aethiopicum. Journal of the American Chemical Society, 133, 2729-2741. http://dx.doi.org/10.1021/ja1101085

[14] Mohamed, A.R. and Mahmound, E.S. (2007) Synthesis and Antitumor Activity of Indolylpyrimidines: Marine Natural Product Meridianin D Analogues. Bioorganic \& Medicinal Chemistry Letters, 15, 1206-1217. http://dx.doi.org/10.1016/j.bmc.2006.11.023

[15] Sofy, A.R., Hmed, A.A., Sharaf, A.M. and El-Dougdoug, K.A. (2014) Structural Changes of Pathogenic Multiple Drug Resistance Bacteria Treated With T. Vulgaris Aqueous Extract. Nature and Science, 12, 83-88.

[16] Caldeira, E.M., Osório, A., Oberosler, E.L., Vaitsman, D.S., Alviano, D.S. and Nojima, M.D.G. (2013) Antimicrobial and Fluoride Release Capacity of Orthodontic Bonding Materials. Journal of Applied Oral Science, 21, 327-334. http://dx.doi.org/10.1590/1678-775720130010

[17] Kaya, E.G., Özbilge, H. and Albayrak, S. (2009) Determination of the Effect of Gentamicin against Staphlococcus aureus by Using Microbroth Kinetic System. ANKEM Dergisi, 23,110-114.

[18] March, S.J. (2000) Advanced Organic Chemistry: Reactions, Mechanisms and Structure. 5th Edition, John Wiley and 
Sons, New York, 1824.

[19] Attaryan, O., Antanosyan, S., Panosyan, G., Asratyan, G. and Matsoyan, S. (2006) Vilsmeier Formyatiom of 3,5-Dimethylpyrazoles. Russian Journal of General Chemistry, 76, 1817-1819. http://dx.doi.org/10.1134/S1070363206110260

[20] El-Nakkady, S.S., Hanna, M.M., Roaiah, H.M. and Ghannam, I.A. (2012) Synthesis, Molecular Docking Study and Antitumor Activity of Novel 2-Phenylindole Derivatives. European Journal of Medicinal Chemistry, 47, 387-398. http://dx.doi.org/10.1016/j.ejmech.2011.11.007

[21] Saundane, A.R., Katkar, V.T. and Vaijinath, V. (2013) Synthesis, Antimicrobial, and Antioxidant Activities of N-[(5'Substituted-2'-phenyl-1H-indol-3-yl)methylene]-5H-dibenzo[b,f]azepine-5-carbohydrazide Derivatives. Journal of Chemistry, 2013, 1- 9. http://dx.doi.org/10.1155/2013/530135

[22] Mahmouda, M.R., El-Ziatya, A.K., Abu El-Azma, F.S.M., Ismail, M.F. and Shibab, S.A. (2013) Utility of Cyano- $N$ (2-oxo-1,2-dihydroindol-3-ylidene)acetohydrazide in the Synthesis of Novel Heterocycles. Journal of Chemical Research, 37, 80-85. http://dx.doi.org/10.3184/174751912X13567100793191

[23] Mohareb, R.M., El-Arab, E.E. and El-Sharkawy, K.A. (2009) The Reaction of Cyanoacetic Acid Hydrazide with 2Acetylfuran: Synthesis of Coumarin, Pyridine, Thiophene and Tthiazole Derivatives with Potential Antimicrobial Activities. Scientia Pharmaceutica, 77; 355-366. http://dx.doi.org/10.3797/scipharm.0901-20

[24] Darwish, E.S., Abdel Fattah, A.M., Attaby, F.A. and Al-Shayea, O.N. (2014) Synthesis and Antimicrobial Evaluation of Some Novel Thiazole, Pyridone, Pyrazole, Chromene, Hydrazone Derivatives Bearing a Biologically Active Sulfonamide Moiety. International Journal of Molecular Sciences, 15, 1237-1254. http://dx.doi.org/10.3390/ijms15011237

[25] Volmajer, J., Toplak, R., Leban, I. and Le Marechal, A.M. (2005) Synthesis of New Iminocoumarins and Their Transformations into N-Chloro and Hydrazono Compounds. Tetrahedron, 61, 7012-7021. http://dx.doi.org/10.1016/j.tet.2005.05.020

[26] Alafeefy, A.M., Alqasoumi, S.I., Ashour, A.E., Masand, V., Al-Jaber, N.A., Hadda, T.B. and Mohamed, M.A. (2012) Quinazoline-Tyrphostin as a New Class of Antitumor Agents, Molecular Properties Prediction, Synthesis and Biological Testing. European Journal of Medicinal Chemistry, 53, 133-140. http://dx.doi.org/10.1016/j.ejmech.2012.03.044

[27] El-Gaml, K.M. (2015) Synthesis and Antimicrobial Evaluation of New Polyfunctionally Substituted Heterocyclic Compounds Derived from 2-Cyano-N-(3-cyanoquinolin-2-yl)acetamide. American Journal of Organic Chemistry, 5, 19.

[28] Ravibabu, V., Janardhan, B., Rajitha, G. and Rajitha, B. (2013) Efficient One-Pot Three-Component Synthesis of Thiazolylpyrazole Derivatives under Conventional Method. Der Chemica Sinica, 4, 79-83.

[29] Dilli Varaprasad, E., Mastan, M. and Sobha Rani, T. (2012) Synthesis and Evaluation of Analgesic Activity of Novel Series of Indole Derivatives Linked to Isoxazole Moiety. Der Pharmacia Lettre, 4, 1431-1437.

[30] El-Nakkady, S., Abbas, S., Roaiah, H. and Ali, I. (2012) Synthesis, Antitumor and Anti-Inflammatory Activities of 2Thienyl-3-Substitued Indole Derivatives. Global Journal of Pharmacology, 6, 166-177.

[31] Chen, Z.-H., Zheng, C.-J., Sun, L.-P. and Piao, H.-R. (2010) Synthesis of New Chalcone Derivatives Containing Rhodanine-3-Acetic Acid Moiety with Potential Anti-Bacterial Activity. European Journal of Medicinal Chemistry, 45, 5739-5743. http://dx.doi.org/10.1016/j.ejmech.2010.09.031

[32] Chaudhari, S.A., Sandeep, R., Patil, S.R., Patil, V.M., Patil, S.V., Jachak, M.N. and Desai, A. (2015) Synthesis of Pyrano[2,3-d]pyridine, Pyrazolo[3,4-b]pyridine Derivatives by Microwave Irradiation and Study of Their Insecticidal Activity. Journal of Chemical and Pharmaceutical Research, 9,476-482.

[33] Shabaan, M., Taher, A.T. and Osma, E.O. (2011) Synthesis of Novel 3,4-Dihydroquinoxalin-2(1H)-one Derivatives. European Journal of Chemistry, 2, 365-371. http://dx.doi.org/10.5155/eurjchem.2.3.365-371.289

[34] Abdelhafez, O.M., Amin, K.M., Batran, R.Z., Maher, T.J., Somaia, A., Nada, S.A. and Sethumadhavan, S. (2010) Synthesis, Anticoagulant and PIVKA-II Induced by New 4-Hydroxycoumarin Derivatives. Bioorganic \& Medicinal Chemistry, 18, 3371-3378. http://dx.doi.org/10.1016/j.bmc.2010.04.009 